\title{
Facile, Direct Reaction of Benzaldehydes to 3-Arylprop-2-Enoic Acids and 3-Arylprop-2-Ynoic Acids in Aqueous Medium
}

\author{
Thies Thiemann ${ }^{*}$, Mohamed W. Elshorbagy', Mostafa H. F. A. Salem², \\ Siraj A. N. Ahmadani'2, Yosef Al-Jasem², Mariam Al Azani' ${ }^{1}$, Mazen A. M. Al-Sulaibi', \\ Bassam Al-Hindawi ${ }^{1}$ \\ ${ }^{1}$ Department of Chemistry, United Arab Emirates University, Al Ain, United Arab Emirates \\ ${ }^{2}$ Department of Chemical Engineering, United Arab Emirates University, Al Ain, United Arab Emirates \\ Email: "thies@uaeu.ac.ae, "thiesthiemann@yahoo.de
}

Received 27 March 2016; accepted 31 May 2016; published 3 June 2016

Copyright (C) 2016 by authors and Scientific Research Publishing Inc.

This work is licensed under the Creative Commons Attribution International License (CC BY).

http://creativecommons.org/licenses/by/4.0/

(c) (i) Open Access

\begin{abstract}
Wittig reactions of benzaldehydes, alkanals, and cycloalkanals as well as of acetophenones are carried out with alkoxycarbonyl methylidenetriphenylphosphoranes in $10 \mathrm{w} \%$ aqueous $\mathrm{NaOH}$, where the cinnamates and alkenoates produced are hydrolysed in situ and the corresponding acids are obtained after mostly simple extractive work-up, often without employing organic solvents. Under the same conditions, benzaldehydes are reacted with alkoxycarbonyl bromomethylidenephosphorane to produce 3-arylprop-2-ynoic acids (arylpropiolic acids).
\end{abstract}

\section{Keywords}

Carboxylic Acids, Arylpropiolic Acids, Wittig Olefination, One Pot Reaction, Aqueous Reaction Medium, Dehydrobromination, Hydrolysis

\section{Introduction}

Cinnamic acids and their derivatives have a wide range of uses. They can have antifungal [1] and/or antimicrobial [2] activity. Applications of cinnamic acid and derivatives can be found in the cosmetic industry, where they have been employed as firming agents in formulations used in skin care products [3]. Additionally, cinnamic acids and derivatives have found use as flavorants [4], and especially the alkoxylated and hydroxylated

${ }^{*}$ Corresponding author.

How to cite this paper: Thiemann, T., Elshorbagy, M.W., Salem, M.H.F.A., Ahmadani, S.A.N., Al-Jasem, Y., Al Azani, M., Al-Sulaibi, M.A.M. and Al-Hindawi, B. (2016) Facile, Direct Reaction of Benzaldehydes to 3-Arylprop-2-Enoic Acids and 3Arylprop-2-Ynoic Acids in Aqueous Medium. International Journal of Organic Chemistry, 6, 126-141. 
cinnamic acids are utilized as stabilizers in the food industry [5]. Also, they are key building blocks in various synthetic preparations in the industry. Cinnamic acid itself is an intermediate in the production of phenylalanine, and thus of aspartame [6]. The authors [7] [8] have communicated their interest in cinnamic acids previously, because some cinnamic acids [7]-[9] and especially dihydroxycinnamic acid derivatives show anti-cancer activity.

Cinnamic acids have been prepared in a number of ways. Among them, the reaction of benzaldehydes with malonic acid is the most common (Knoevenagel reaction with subsequent decarboxylation [10]-[11]). This strategy has been augmented recently by the reaction of benzaldehydes with Meldrum's acid [12]. Also, cinnamic acids can be prepared by the reaction of benzaldehydes with acetic anhydride/sodium acetate (Perkin reaction [13]), and of iodoarenes with acrylates via Heck reaction [14] with subsequent hydrolysis in a two-step sequence. Some of the reactions above give variable yields and others require special catalysts [12]. Therefore, the Wittig olefination reaction of benzaldehydes with stabilized alkoxymethylidenetriphenylphosphoranes remains an alternative in the preparation of cinnamic acids, and so has been used extensively, too [15].

Conventional Wittig reactions with stabilized phosphoranes have been carried under elevated temperature in organic solvents such as THF and benzene, sometimes under acid catalysis (Bestmann variation) [16]. In more recent times, so-called non-classical reaction conditions [17] have been forwarded for the Wittig olefination, such as carrying out reactions under solventless [18] conditions or in ionic liquids [19]. Often, this has been combined with microwave irradiation or ultrasonication. Also, water soluble phosphoranes [20]-[22] have been developed to carry out the reactions in aqueous solutions. Afterwards, it was seen that olefination reactions could also be performed in water with stabilized and semi-stabilized, non-water soluble phosphoranes [23]-[34], with one communication on a Wittig reaction utilizing semi-stabilized and non-stabilized phosphoranes in a biphasic mixture under phase transfer conditions (PTC) [35]. Nevertheless, almost all of these cases involve extractive work-up and often chromatographic separations using organic solvents. Mostly, such work-up utilizes greater amounts of organic solvent than does the reaction itself, if it were run in organic medium. Before, the authors had developed a biphasic reaction system of water and hexane for the Wittig olefination with stabilized and semi-stabilized phosphoranes [36]. Here, the reaction proceeds mostly at the solvent interface [37], giving the product after simple evaporation of the hexane phase, where the organic solvent can be recycled. Nevertheless, possibilities of using such a strategy are rare.

In the following, a methodology is introduced which involves a one-pot olefination-hydrolysis sequence, where the unsaturated carboxylic acid is the final product, which can be isolated by acidification of the reaction medium, and, in the case of the cinnamic acid products, by simple filtration. The reaction sequence is wellsuited for educational laboratories also, and has been shown to give reproducible good yields with our undergraduate students.

\section{Experimental}

\subsection{Chemicals and Instruments}

\section{Experimental}

General Remarks. Melting points were measured on a Stuart SMP 10 melting point apparatus and are uncorrected. Infrared spectra were measured with a Thermo/Nicolet Nexus $470 \mathrm{FT}$-IR ESP spectrometer. ${ }^{1} \mathrm{H}$ and ${ }^{13} \mathrm{C}$ NMR spectra were recorded with a Varian $400 \mathrm{NMR}$ spectrometer $\left({ }^{1} \mathrm{H}\right.$ at $395.7 \mathrm{MHz},{ }^{13} \mathrm{C}$ at $\left.100.5 \mathrm{MHz}\right)$. The assignments of the carbon signals were aided by DEPT 90 and DEPT 135 experiments (DEPT $=$ Distortionless Enhancement by Polarisation Transfer). The chemical shifts are relative to TMS (solvent $\mathrm{CDCl}_{3}$, unless otherwise noted). Mass spectra were measured with a JMS-01-SG-2 spectrometer, and with an Agilent QTOF 6540 UHD. Column chromatography, where necessary, was performed on silica gel ( $\mathrm{S}, 0.063 \mathrm{~mm}-0.1 \mathrm{~mm}$, Riedel de Haen and Merck grade 9385).

2-Methoxybenzaldehyde (1a), 2,4-dimethoxybenzaldehyde (1g) and 4-benzyloxybenzaldehyde (1x) were prepared from the corresponding, commercially available hydroxybenzaldehydes $\left(\mathrm{KOH}, \mathrm{DMSO}, \mathrm{CH}_{3} \mathrm{I}\right.$ or $\mathrm{PhCH}_{2} \mathrm{Br}$ ) [38]. Benzaldehydes $\mathbf{1 n}$ and $\mathbf{1 0}$ were synthesized by bromination (NBS, DMF, rt) of 2-hydroxy- and 4-hydroxybenzaldehydes, respectively, followed by alkylation $\left(\mathrm{KOH}, \mathrm{DMSO}, \mathrm{CH}_{3} \mathrm{I}\right)$. The other benzaldehydes were acquired commercially. The 4-haloacetophenones $7 \mathbf{b}-\mathbf{d}$ and 1 -acetylanthracene (8) were obtained by acetylation $\left(\mathrm{AcCl}, \mathrm{AlCl}_{3}\right)$ of the parent acetophenones and anthracene, respectively. Phosphoranes 2a [39] and 2b [40] were prepared according to literature procedures. 
20 and 22 were prepared by Wittig olefination, starting from 2-benzyloxybenzaldehyde and benzoylmethylidenetriphenylphosphorane and from 2-benzyloxycinnamaldehyde (24) and toluoylmethylidenetriphenylphosphorane.

\subsection{General Procedure A}

(E)-Cinnamic acid (3f). To a mixture of benzaldehyde (1f, $1.60 \mathrm{~g}, 15.1 \mathrm{mmol})$ and ethoxycarbonylmethylidenetriphenylphosphorane (2a, $6.82 \mathrm{~g}, 19.5 \mathrm{mmmol})$ was given an aq. $\mathrm{NaOH}$ solution $(9.1 \mathrm{w} \%, 3.5 \mathrm{~g}(87.5 \mathrm{mmol})$ $\mathrm{NaOH}$ in $35 \mathrm{~mL} \mathrm{H} \mathrm{H}_{2} \mathrm{O}$ ) and the resulting suspension was stirred at $75^{\circ} \mathrm{C}$ for $23 \mathrm{~h}$. Triphenylphosphine oxide was filtered off the cooled solution. Thereafter, the filtrate was acidified carefully with $15 \mathrm{w} \%$ aq. $\mathrm{HCl}$. The resulting suspension was cooled and filtered. The solid obtained was dried in air to give cinnamic acid (3f, $1.89 \mathrm{~g}, 77 \%)$ as a colorless solid; mp. $132^{\circ} \mathrm{C}$ [Lit. $\left.132^{\circ} \mathrm{C}-135^{\circ} \mathrm{C}[41]\right] ; v_{\max }\left(\mathrm{KBr} / \mathrm{cm}^{-1}\right) 1690(\mathrm{C}=\mathrm{O}), 1631,1454,1312,1290$, $1225,771,714 ; \delta_{\mathrm{H}}\left(270 \mathrm{MHz}, \mathrm{DMSO}-\mathrm{d}^{6}\right) 6.51\left(1 \mathrm{H}, \mathrm{d},{ }^{3} \mathrm{~J}=16.0 \mathrm{~Hz}\right), 7.39(2 \mathrm{H}, \mathrm{m}), 7.57\left(1 \mathrm{H}, \mathrm{d},{ }^{3} \mathrm{~J}=16.0 \mathrm{~Hz}\right)$, 7.59 - $7.66(3 \mathrm{H}, \mathrm{m}), 12.35(1 \mathrm{H}, \mathrm{bs}) ; \delta_{\mathrm{C}}\left(67.8 \mathrm{MHz}, \mathrm{DMSO}-\mathrm{d}^{6}\right)$ 119.7, $128.6(2 \mathrm{C}), 129.3$ (2C), 130.7, 134.7, 144.4, 168.0; MS (EI, $70 \mathrm{eV}) \mathrm{m} / \mathrm{z}(\%) 148\left(\mathrm{M}^{+}\right)$(100), 77 (40).

(E)-2-Methoxycinnamic acid (3a) (Procedure A). Colorless solid; mp. $187^{\circ} \mathrm{C}$ [Lit. $\left.183^{\circ} \mathrm{C}-186^{\circ} \mathrm{C}[41]\right]$; $v_{\max }$ $\left(\mathrm{KBr} / \mathrm{cm}^{-1}\right) 1682(\mathrm{C}=\mathrm{O}), 1620(\mathrm{C}=\mathrm{C}), 1489,1463,1426,1332,1282,1248,1220(\mathrm{C}-\mathrm{O}), 1025,994,941,755$, 696, 596, 567; $\delta_{\mathrm{H}}\left(400 \mathrm{MHz}, \mathrm{DMSO}-\mathrm{d}^{6}\right) 3.84\left(3 \mathrm{H}, \mathrm{s}, \mathrm{OCH}_{3}\right), 6.48\left(1 \mathrm{H}, \mathrm{d},{ }^{3} \mathrm{~J}=16.0 \mathrm{~Hz}\right), 6.96\left(1 \mathrm{H}, \mathrm{dd},{ }^{3} \mathrm{~J}=7.6\right.$ $\left.\mathrm{Hz},{ }^{3} J=7.2 \mathrm{~Hz}\right), 7.06\left(1 \mathrm{H}, \mathrm{d},{ }^{3} J=8.4 \mathrm{~Hz}\right), 7.36-7.40(1 \mathrm{H}, \mathrm{m}), 7.64-7.66(1 \mathrm{H}, \mathrm{m}), 7.81\left(1 \mathrm{H}, \mathrm{d},{ }^{3} \mathrm{~J}=16.0 \mathrm{~Hz}\right)$; $\delta_{\mathrm{C}}\left(100.5 \mathrm{MHz}, \mathrm{DMSO}^{6} \mathrm{~d}^{6}\right) 56.1,112.2,119.7,121.2,122.9,128.9,132.2,139.1,158.2,168.3$; MS (EI, $\left.70 \mathrm{eV}\right)$ $m / z(\%)=178\left(\mathrm{M}^{+}, 89\right), 147\left(\mathrm{M}^{+}-\mathrm{OCH}_{3}, 100\right)$.

(E)-4-Methoxycinnamic acid (3b) (Procedure A). Colorless solid; mp. $173^{\circ} \mathrm{C}$ [Lit. $173^{\circ} \mathrm{C}$ [41]]; $v_{\max }(\mathrm{KBr} /$ $\left.\mathrm{cm}^{-1}\right) 1694(\mathrm{C}=\mathrm{O}), 1596(\mathrm{C}=\mathrm{C}), 1336,826,685,566,527,513 ; \delta_{\mathrm{H}}\left(400 \mathrm{MHz}, \mathrm{CDCl}_{3}\right) 3.85\left(3 \mathrm{H}, \mathrm{s}, \mathrm{OCH}_{3}\right), 6.33$ $\left(1 \mathrm{H}, \mathrm{d},{ }^{3} J=16.0 \mathrm{~Hz}\right), 6.92\left(2 \mathrm{H}, \mathrm{d},{ }^{3} J=8.8 \mathrm{~Hz}\right), 7.51\left(2 \mathrm{H}, \mathrm{d},{ }^{3} J=8.8 \mathrm{~Hz}\right), 7.75\left(1 \mathrm{H}, \mathrm{d},{ }^{3} J=16.0 \mathrm{~Hz}\right) ; \delta_{\mathrm{C}}(100.5$ $\left.\mathrm{MHz}, \mathrm{CDCl}_{3}\right) 55.4\left(\mathrm{OCH}_{3}\right), 114.4(2 \mathrm{C}, \mathrm{CH}), 114.6(\mathrm{CH}), 126.8\left(\mathrm{C}_{\text {quat }}\right), 130.1(2 \mathrm{C}, \mathrm{CH}), 146.7(\mathrm{CH}), 161.7$ $\left(\mathrm{C}_{\text {quat }}\right), 172.2\left(\mathrm{C}_{\text {quat }}, \mathrm{CO}\right)$; $\mathrm{MS}(70 \mathrm{eV}, \mathrm{EI}) \mathrm{m} / z(\%) 178\left(\mathrm{M}^{+}, 65\right)$.

(E)-4-Ethoxycinnamic acid (3c) (Procedure A). Colorless solid, mp. $196^{\circ} \mathrm{C}$ [Lit. mp. $\left.195^{\circ} \mathrm{C}-199^{\circ} \mathrm{C}[41]\right] ; \delta_{\mathrm{H}}$ (400 MHz, DMSO-d $\left.{ }^{6}\right) 1.30\left(3 \mathrm{H}, \mathrm{t},{ }^{3} J=7.2 \mathrm{~Hz}, \mathrm{CH}_{3}\right), 4.02\left(2 \mathrm{H}, \mathrm{q},{ }^{3} J=7.2 \mathrm{~Hz}, \mathrm{OCH}_{2}\right), 6.34\left(1 \mathrm{H}, \mathrm{d},{ }^{3} J=16.0\right.$ $\mathrm{Hz}), 6.92\left(2 \mathrm{H}, \mathrm{d},{ }^{3} J=8.4 \mathrm{~Hz}\right), 7.51\left(1 \mathrm{H}, \mathrm{d},{ }^{3} \mathrm{~J}=16.0 \mathrm{~Hz}\right), 7.59\left(2 \mathrm{H}, \mathrm{d},{ }^{3} J=8.4 \mathrm{~Hz}\right) ; \delta_{\mathrm{C}}\left(100.5 \mathrm{MHz}, \mathrm{DMSO}-\mathrm{d}^{6}\right)$ $15.0\left(\mathrm{CH}_{3}\right), 63.7\left(\mathrm{CH}_{2}\right), 115.2(2 \mathrm{C}, \mathrm{CH}), 116.8(\mathrm{CH}), 127.1\left(\mathrm{C}_{\text {quat }}\right), 130.4(2 \mathrm{C}, \mathrm{CH}), 144.2(\mathrm{CH}), 160.7\left(\mathrm{C}_{\text {quat }}\right)$, $168.3\left(\mathrm{C}_{\text {quat }}, \mathrm{CO}\right)$; MS (EI, $\left.70 \mathrm{eV}\right) \mathrm{m} / z(\%)=192\left(\mathrm{M}^{+}, 61\right), 146(54), 118(100)$.

(E)-4'-Propoxycinnamic acid (3d) (Procedure A). Colorless solid, mp. $155^{\circ} \mathrm{C}$ [Lit. $\left.155^{\circ} \mathrm{C}[42]\right] ; \delta_{\mathrm{H}}(400$ $\left.\mathrm{MHz}, \mathrm{DMSO}-\mathrm{d}^{6}\right) 0.96\left(3 \mathrm{H}, \mathrm{t},{ }^{3} \mathrm{~J}=7.2 \mathrm{~Hz}, \mathrm{CH}_{3}\right), 1.70\left(\mathrm{qt},{ }^{3} \mathrm{~J}=7.2 \mathrm{~Hz},{ }^{3} \mathrm{~J}=6.4 \mathrm{~Hz}\right), 3.95\left(2 \mathrm{H}, \mathrm{t},{ }^{3} \mathrm{~J}=6.4 \mathrm{~Hz}\right)$, $6.34\left(1 \mathrm{H}, \mathrm{d},{ }^{3} \mathrm{~J}=16.0 \mathrm{~Hz}\right), 6.94\left(2 \mathrm{H}, \mathrm{d},{ }^{3} J=8.8 \mathrm{~Hz}\right), 7.51\left(1 \mathrm{H}, \mathrm{d},{ }^{3} J=16.0 \mathrm{~Hz}\right), 7.59\left(2 \mathrm{H}, \mathrm{d},{ }^{3} J=8.8 \mathrm{~Hz}\right) ; \delta_{\mathrm{C}}$ (100.5 MHz, DMSO-d $\left.{ }^{6}\right)$ 14.1, 19.1, 67.8, 115.2 (2C), 116.8, 127.1, 130.4, 144.2, 160.9, 168.3.

(E)-4' -(N,N-Dimethylamino)cinnamic acid (3e) (Procedure A). Colorless solid, mp. 226 C; [Lit. $227-228^{\circ} \mathrm{C}$ [41]]; $v_{\max }\left(\mathrm{KBr} / \mathrm{cm}^{-1}\right) 2922(\mathrm{CH}), 1679(\mathrm{C}=\mathrm{O}), 1598(\mathrm{C}=\mathrm{C}), 1230,1202,815 ; \delta_{\mathrm{H}}\left(400 \mathrm{MHz}, \mathrm{DMSO}-\mathrm{d}^{6}\right) 2.94(6 \mathrm{H}$, $\left.\mathrm{s}, 2 \mathrm{CH}_{3}\right), 6.19\left(1 \mathrm{H}, \mathrm{d},{ }^{3} J=16.0 \mathrm{~Hz}\right), 6.68\left(2 \mathrm{H}, \mathrm{d},{ }^{3} J=9.2 \mathrm{~Hz}\right), 7.43\left(1 \mathrm{H}, \mathrm{d},{ }^{3} J=16.0 \mathrm{~Hz}\right), 7.45\left(2 \mathrm{H}, \mathrm{d},{ }^{3} J=9.2\right.$ $\mathrm{Hz}), 11.93(\mathrm{bs}, 1 \mathrm{H}, \mathrm{OH}) ; \delta_{\mathrm{C}}\left(100.5 \mathrm{MHz}, \mathrm{CDCl}_{3}\right) 39.4\left(2 \mathrm{C}, \mathrm{CH}_{3}\right), 111.8(2 \mathrm{C}, \mathrm{CH}), 113.1(\mathrm{CH}), 121.6\left(\mathrm{C}_{\text {quat }}\right)$, $129.6(2 \mathrm{C}, \mathrm{CH}), 144.6(\mathrm{CH}), 151.6\left(\mathrm{C}_{\text {quat }}\right), 168.0\left(\mathrm{C}_{\text {quat }}, \mathrm{CO}\right)$; $\mathrm{MS}(\mathrm{EI}, 70 \mathrm{eV}) \mathrm{m} / \mathrm{z}(\%) 191\left(\mathrm{M}^{+}, 100\right), 147(17)$.

(E)-2,4-Dimethoxycinnamic acid (3g) (Procedure A). Colorless solid, mp. $191^{\circ} \mathrm{C}$; [Lit. $192^{\circ} \mathrm{C}-194^{\circ} \mathrm{C}[41]$; $\delta_{\mathrm{H}}\left(400 \mathrm{MHz}, \mathrm{DMSO}-\mathrm{d}^{6} / \mathrm{CDCl}_{3}\right) 3.81\left(3 \mathrm{H}, \mathrm{s}, \mathrm{OCH}_{3}\right), 3.86\left(3 \mathrm{H}, \mathrm{s}, \mathrm{OCH}_{3}\right), 6.33\left(1 \mathrm{H}, \mathrm{d},{ }^{3} \mathrm{~J}=16.0 \mathrm{~Hz}\right), 6.51(2 \mathrm{H}$, $\mathrm{m}), 7.47\left(1 \mathrm{H}, \mathrm{d},{ }^{3} \mathrm{~J}=8.0 \mathrm{~Hz}\right), 7.80\left(1 \mathrm{H}, \mathrm{d},{ }^{3} \mathrm{~J}=16.0 \mathrm{~Hz}\right) ; \delta_{\mathrm{H}}\left(100.5 \mathrm{MHz}, \mathrm{DMSO}-\mathrm{d}^{6} / \mathrm{CDCl}_{3}\right) 55.2\left(\mathrm{OCH}_{3}\right), 55.3$ $\left(\mathrm{OCH}_{3}\right), 98.1(\mathrm{CH}), 105.9(\mathrm{CH}), 115.9\left(\mathrm{C}_{\text {quat }}\right), 116.3(\mathrm{CH}), 129.6(\mathrm{CH}), 138.8(\mathrm{CH}), 159.4\left(\mathrm{C}_{\text {quat }}\right), 162.7\left(\mathrm{C}_{\text {quat }}\right)$, $168.3\left(\mathrm{C}_{\text {quat }}, \mathrm{CO}\right)$.

(E)-2,5-Dimethoxycinnamic acid (3h) (Procedure A). Colorless solid; mp. $149{ }^{\circ} \mathrm{C}$; [Lit. $148^{\circ} \mathrm{C}-150^{\circ} \mathrm{C}[41]$; $v_{\max }\left(\mathrm{KBr} / \mathrm{cm}^{-1}\right) 1697(\mathrm{C}=\mathrm{O}), 1627(\mathrm{C}=\mathrm{C}), 1581,1503,1430,1323,1228(\mathrm{C}-\mathrm{O}), 1189(\mathrm{C}-\mathrm{O}), 1052,1019,988$, $941,858,788,700,629,537,487,456 ; \delta_{\mathrm{H}}\left(400 \mathrm{MHz}, \mathrm{DMSO}-\mathrm{d}^{6}\right) 3.72\left(3 \mathrm{H}, \mathrm{s}, \mathrm{OCH}_{3}\right), 3.78\left(3 \mathrm{H}, \mathrm{s}, \mathrm{OCH}_{3}\right), 6.53$ $\left(1 \mathrm{H}, \mathrm{d},{ }^{3} J=16.4 \mathrm{~Hz}\right), 6.97\left(1 \mathrm{H}, \mathrm{d},{ }^{3} J=6.4 \mathrm{~Hz}\right), 6.99\left(1 \mathrm{H}, \mathrm{d},{ }^{3} J=6.4 \mathrm{~Hz}\right), 7.21\left(1 \mathrm{H}, \mathrm{d},{ }^{4} J=3.2 \mathrm{~Hz}\right), 7.78(1 \mathrm{H}, \mathrm{d}$, $\left.{ }^{3} J=16.4 \mathrm{~Hz}\right) ; \delta_{\mathrm{C}}\left(100.5 \mathrm{MHz}, \mathrm{CDCl}_{3}\right) 56.0\left(\mathrm{OCH}_{3}\right), 57.0\left(\mathrm{OCH}_{3}\right), 112.5,113.5,117.8,118.0,123.6,142.2$, $153.2\left(\mathrm{C}_{\text {quat }}\right), 153.6\left(\mathrm{C}_{\text {quat }}\right), 173.0\left(\mathrm{C}_{\text {quat }}, \mathrm{CO}\right)$; $\mathrm{MS}(\mathrm{EI}, 70 \mathrm{eV}) \mathrm{m} / z(\%)=208\left(\mathrm{M}^{+}, 100\right), 177\left(\mathrm{M}^{+}-\mathrm{OCH}_{3}, 33\right)$.

4-Bromo-2,5-dimethoxycinnamic acid (3i) (Procedure $\mathrm{A}$ ) as a mixture of $E$ - and $Z$-isomers (72/28), pale yellow solid, mp. $177^{\circ} \mathrm{C}-180^{\circ} \mathrm{C}$ (for the $E$-/Z-mixture); $v_{\max }\left(\mathrm{KBr} / \mathrm{cm}^{-1}\right.$ ) [E/Z-mixture] $3502-2829$ (bs, $\mathrm{OH}$ ), 
$1687(\mathrm{C}=\mathrm{O}), 1677(\mathrm{C}=\mathrm{O}), 1629(\mathrm{C}=\mathrm{C}), 1565,1499,1441,1392,1329,1255,1207,1052,987,917,867,822$, 739, 696, 638, 617, 541; (Z)-isomer $\delta_{\mathrm{H}}\left(400 \mathrm{MHz}_{\mathrm{CDCl}}\right) 3.80\left(3 \mathrm{H}, \mathrm{s}, \mathrm{OCH}_{3}\right), 3.84\left(3 \mathrm{H}, \mathrm{s}, \mathrm{OCH}_{3}\right), 6.01(1 \mathrm{H}, \mathrm{d}$, $\left.{ }^{3} J=12.8 \mathrm{~Hz}\right), 7.07(1 \mathrm{H}, \mathrm{s}), 7.23\left(1 \mathrm{H}, \mathrm{d},{ }^{3} J=12.8 \mathrm{~Hz}\right), 7.39(1 \mathrm{H}, \mathrm{s}) ; \delta_{\mathrm{C}}\left(100.5 \mathrm{MHz}, \mathrm{CDCl}_{3}\right) 56.2\left(\mathrm{OCH}_{3}\right), 56.3$ $\left(\mathrm{OCH}_{3}\right), 113.5\left(\mathrm{C}_{\text {quat }}\right), 114.8(\mathrm{CH}), 115.8(\mathrm{CH}), 119.0(\mathrm{CH}), 123.0\left(\mathrm{C}_{\text {quat }}\right), 140.4(\mathrm{CH}), 149.3\left(\mathrm{C}_{\text {quat }}\right), 151.8$ $\left(\mathrm{C}_{\text {quat }}\right), 170.9\left(\mathrm{C}_{\text {quat }}, \mathrm{CO}\right) ;(E)$-isomer $\delta_{\mathrm{H}}\left(400 \mathrm{MHz}, \mathrm{CDCl}_{3}\right) 3.85\left(3 \mathrm{H}, \mathrm{s}, \mathrm{OCH}_{3}\right), 3.89\left(3 \mathrm{H}, \mathrm{s}, \mathrm{OCH}_{3}\right), 6.51(1 \mathrm{H}$, d, $\left.{ }^{3} J=16.0 \mathrm{~Hz}\right), 7.03(1 \mathrm{H}, \mathrm{s}), 7.13(1 \mathrm{H}, \mathrm{s}), 8.00\left(1 \mathrm{H}, \mathrm{d},{ }^{3} J=16.0 \mathrm{~Hz}\right) ; \delta_{\mathrm{C}}\left(100.5 \mathrm{MHz}, \mathrm{CDCl}_{3}\right) 56.3\left(\mathrm{OCH}_{3}\right)$, $56.8\left(\mathrm{OCH}_{3}\right), 111.6(\mathrm{CH}), 115.3\left(\mathrm{C}_{\text {quat }}\right), 116.9(\mathrm{CH}), 118.0(\mathrm{CH}), 122.6\left(\mathrm{C}_{\text {quat }}\right), 141.5(\mathrm{CH}), 150.2\left(\mathrm{C}_{\text {quat }}\right), 153.0$ $\left(\mathrm{C}_{\text {quat }}\right), 172.2\left(\mathrm{C}_{\text {quat }}, \mathrm{CO}\right)$; MS (EI, $\left.70 \mathrm{eV}\right) \mathrm{m} / z(\%)=288\left(\left[{ }^{81} \mathrm{BrM}^{+}, 11\right), 286\left(\left[{ }^{79} \mathrm{BrM}^{+}, 13\right)\right.\right.$.

(E)-4-Methylcinnamic acid (3j) (Procedure A). Colorless solid, mp. $195^{\circ} \mathrm{C}-199^{\circ} \mathrm{C}$ [Lit. $196^{\circ} \mathrm{C}-198^{\circ} \mathrm{C}[41]$; $v_{\max }\left(\mathrm{KBr} / \mathrm{cm}^{-1}\right) 3550-2330(\mathrm{bs}, \mathrm{OH}), 1678(\mathrm{C}=\mathrm{O}), 1624(\mathrm{C}=\mathrm{C}), 1423,1312,1285,1223,987,944,812,687$, 554,$495 ; \delta_{\mathrm{H}}\left(400 \mathrm{MHz}, \mathrm{CDCl}_{3}\right) 2.38\left(3 \mathrm{H}, \mathrm{s}, \mathrm{CH}_{3}\right), 6.40\left(1 \mathrm{H}, \mathrm{d},{ }^{3} \mathrm{~J}=16.0 \mathrm{~Hz}\right), 7.21\left(2 \mathrm{H}, \mathrm{d},{ }^{3} \mathrm{~J}=8.0 \mathrm{~Hz}\right), 7.45$ $\left(2 \mathrm{H}, \mathrm{d},{ }^{3} \mathrm{~J}=8.0 \mathrm{~Hz}\right), 7.76\left(1 \mathrm{H}, \mathrm{d},{ }^{3} J=16.0 \mathrm{~Hz}\right) ; \delta_{\mathrm{H}}\left(400 \mathrm{MHz}, \mathrm{DMSO}-\mathrm{d}^{6}\right) 2.33\left(3 \mathrm{H}, \mathrm{s}, \mathrm{CH}_{3}\right), 6.49\left(1 \mathrm{H}, \mathrm{d},{ }^{3} J=\right.$ $16.0 \mathrm{~Hz}), 7.23\left(2 \mathrm{H}, \mathrm{d},{ }^{3} J=8.4 \mathrm{~Hz}\right), 7.57\left(2 \mathrm{H}, \mathrm{d},{ }^{3} J=8.4 \mathrm{~Hz}\right), 7.58\left(1 \mathrm{H}, \mathrm{d},{ }^{3} J=16.0 \mathrm{~Hz}\right), 12.3(1 \mathrm{H}, \mathrm{bs}, \mathrm{OH}) ; \delta_{\mathrm{C}}$ $\left(100.5 \mathrm{MHz}, \mathrm{DMSO}-\mathrm{d}^{6}\right) 30.0\left(\mathrm{CH}_{3}\right), 118.1(\mathrm{CH}), 128.2(2 \mathrm{C}, \mathrm{CH}), 129.6(2 \mathrm{C}, \mathrm{CH}), 131.6\left(\mathrm{C}_{\text {quat }}\right), 140.2\left(\mathrm{C}_{\text {quat }}\right)$, $144.0(\mathrm{CH}), 167.6\left(\mathrm{C}_{\text {quat }}, \mathrm{CO}\right)$; $\mathrm{MS}(\mathrm{EI}, 70 \mathrm{eV}) \mathrm{m} / \mathrm{z}(\%)=162(100)\left(\mathrm{M}^{+}\right), 115(43)$.

(E)-3-Chlorocinnamic acid (3k) (Procedure A). Colorless crystals, mp. $165^{\circ} \mathrm{C}$; [Lit. $161^{\circ} \mathrm{C}-164^{\circ} \mathrm{C}[41]$ ]; $v_{\max }\left(\mathrm{KBr} / \mathrm{cm}^{-1}\right) 1677(\mathrm{C}=\mathrm{O}), 1632(\mathrm{C}=\mathrm{C}), 1430,1323,1301,1283,1230,1200,943,864,727,680,669,597$, $550 ; \delta_{\mathrm{H}}\left(400 \mathrm{MHz}, \mathrm{DMSO}-\mathrm{d}^{6}\right) 6.59\left(1 \mathrm{H}, \mathrm{d},{ }^{3} \mathrm{~J}=16.4 \mathrm{~Hz}\right), 7.41-7.45(2 \mathrm{H}, \mathrm{m}), 7.55\left(1 \mathrm{H}, \mathrm{d},{ }^{3} J=16.4 \mathrm{~Hz}\right), 7.63-$ $7.65(1 \mathrm{H}, \mathrm{m}), 7.78$ - $7.79(1 \mathrm{H}, \mathrm{m}) ;{ }^{13} \mathrm{C}$ NMR (100.5 MHz, DMSO-d $\left.{ }^{6}\right)$ 121.4, 127.2, 128.3, 130.2, 131.1, 134.2, 137.0, 142.7, 167.8; MS (EI, $70 \mathrm{eV}) m / z(\%)=182\left(\left[{ }^{81} \mathrm{Cl}^{\prime} \mathrm{M}^{+}, 100\right), 181\left(\left[{ }^{79} \mathrm{ClM}^{+}\right], 77\right), 147\right.$ (36).

3-Bromocinnamic acid (3L) (Procedure A). Colorless solid; mp. $170^{\circ} \mathrm{C}$ [Lit. $\left.177^{\circ} \mathrm{C}[41]\right] ; v_{\max }\left(\mathrm{KBr} / \mathrm{cm}^{-1}\right)$ 3050 - 2550 (bs, OH), $2972(\mathrm{C}-\mathrm{H}), 1694(\mathrm{C}=\mathrm{O}), 1629(\mathrm{C}=\mathrm{C}), 1424,1318,1219(\mathrm{C}-\mathrm{O}), 977,936,784,720,664$, $542 ; \delta_{\mathrm{H}}\left(400 \mathrm{MHz}, \mathrm{DMSO}-\mathrm{d}^{6}\right) 6.57\left(1 \mathrm{H}, \mathrm{d},{ }^{3} \mathrm{~J}=16.4 \mathrm{~Hz}\right), 7.35\left(1 \mathrm{H}, \mathrm{dd},{ }^{3} J=8.0 \mathrm{~Hz},{ }^{3} \mathrm{~J}=8.0 \mathrm{~Hz}\right), 7.52-7.62$ $(2 \mathrm{H}, \mathrm{m}), 7.52\left(1 \mathrm{H}, \mathrm{d},{ }^{3} J=16.4 \mathrm{~Hz}\right), 7.67\left(1 \mathrm{H}, \mathrm{bd},{ }^{3} J=8.0 \mathrm{~Hz}\right), 7.89(1 \mathrm{H}, \mathrm{m}) ; \delta_{\mathrm{C}}\left(100.5 \mathrm{MHz}, \mathrm{DMSO}-\mathrm{d}^{6}\right) 121.3$, 122.7, 127.5, 131.1, 131.4, 133.2, 137.2, 142.7, 167.8 (CO); MS (EI, $70 \mathrm{eV}) \mathrm{m} / z(\%)=228(95)\left(\left[{ }^{81} \mathrm{Br}\right] \mathrm{M}^{+}\right), 226$ (100) $\left(\left[{ }^{79} \mathrm{Br}\right] \mathrm{M}^{+}\right), 147(61)$.

(E)-3-Fluorocinnamic acid (3m) (Procedure A).Colorless solid; mp. $168^{\circ} \mathrm{C}-169^{\circ} \mathrm{C}$ [Lit. $167^{\circ} \mathrm{C}-170^{\circ} \mathrm{C}$ [41]]; $v_{\max }\left(\mathrm{KBr} / \mathrm{cm}^{-1}\right) 1693(\mathrm{C}=\mathrm{O}), 1632,1588,1295,1249,940,859,782,552 ; \delta_{\mathrm{H}}\left(400 \mathrm{MHz}, \mathrm{DMSO}-\mathrm{d}^{6}\right) 6.57$ $\left(1 \mathrm{H}, \mathrm{d},{ }^{3} J=16.0 \mathrm{~Hz}\right), 7.19-7.24(1 \mathrm{H}, \mathrm{m}), 7.40-7.56(3 \mathrm{H}, \mathrm{m}), 7.50\left(1 \mathrm{H}, \mathrm{d},{ }^{3} J=16.0 \mathrm{~Hz}\right) ; \delta_{\mathrm{C}}(100.5 \mathrm{MHz}$ DMSO-d $\left.{ }^{6}\right) 114.8\left({ }^{2} J_{\mathrm{CF}}=21.7 \mathrm{~Hz}\right), 117.3\left({ }^{2} J_{\mathrm{CF}}=21.0 \mathrm{~Hz}\right), 121.3,125.1,131.3\left({ }^{3} J_{\mathrm{CF}}=8.2 \mathrm{~Hz}\right), 137.2\left({ }^{3} J_{\mathrm{CF}}=8.2\right.$ $\mathrm{Hz}), 143.0,162.8\left({ }^{1} J_{\mathrm{CF}}=243 \mathrm{~Hz}\right), 167.9$ (CO); MS (EI, $\left.70 \mathrm{eV}\right) \mathrm{m} / z(\%)=166(100)\left(\mathrm{M}^{+}\right), 149(30), 121(33)$.

(E)-3,5-Dibromo-4-methoxycinnamic acid (3n) [43] (Procedure A). Colorless solid; mp. $198^{\circ} \mathrm{C} . v_{\max }(\mathrm{KBr} /$ $\left.\mathrm{cm}^{-1}\right) 3100-2597(\mathrm{bs}, \mathrm{OH}), 1703(\mathrm{C}=\mathrm{O}), 1633(\mathrm{C}=\mathrm{C}), 1472,1424,1268,1217,978,851,747 ; \delta_{\mathrm{H}}(400 \mathrm{MHz}$ $\left.\mathrm{CDCl}_{3}\right) 3.92\left(3 \mathrm{H}, \mathrm{s}, \mathrm{OCH}_{3}\right), 6.37\left(1 \mathrm{H}, \mathrm{d},{ }^{3} J=16.4 \mathrm{~Hz}\right), 7.59\left(1 \mathrm{H}, \mathrm{d},{ }^{3} \mathrm{~J}=16.4 \mathrm{~Hz}\right), 7.69(2 \mathrm{H}, \mathrm{s}) ; \delta_{\mathrm{C}}(100.5 \mathrm{MHz}$ $\left.\mathrm{CDCl}_{3}\right) 60.8\left(\mathrm{OCH}_{3}\right), 118.8\left(2 \mathrm{C}, \mathrm{C}_{\text {quat }}\right), 119.0(\mathrm{CH}), 132.3(2 \mathrm{C}, \mathrm{CH}), 143.3(\mathrm{CH}), 155.8\left(\mathrm{C}_{\text {quat }}\right), 170.9\left(\mathrm{C}_{\text {quat }}\right.$, $\mathrm{CO})$.

(E)-2-Bromo-4,5-dimethoxycinnamic acid (3o) (Procedure A).Colorless solid, mp. $240^{\circ} \mathrm{C}-244^{\circ} \mathrm{C}$ [Lit. $\left.246^{\circ} \mathrm{C}-247^{\circ} \mathrm{C}[44]\right] ; v_{\max }\left(\mathrm{KBr} / \mathrm{cm}^{-1}\right) 3496-2578(\mathrm{bs}, \mathrm{OH}), 1697(\mathrm{C}=\mathrm{O}), 1596(\mathrm{C}=\mathrm{C}), 1512,1390,1310,1267$, $1206,1163,1027,969,854,814 ; \delta_{\mathrm{H}}\left(400 \mathrm{MHz} \mathrm{CDCl}_{3}\right) 3.87\left(3 \mathrm{H}, \mathrm{s}, \mathrm{OCH}_{3}\right), 3.90\left(3 \mathrm{H}, \mathrm{s}, \mathrm{OCH}_{3}\right), 6.55\left(1 \mathrm{H}, \mathrm{d},{ }^{3} J\right.$ $=16.0 \mathrm{~Hz}), 6.58(1 \mathrm{H}, \mathrm{s}), 6.77(1 \mathrm{H}, \mathrm{s}), 7.60\left(1 \mathrm{H}, \mathrm{d},{ }^{3} J=16.0 \mathrm{~Hz}\right) ; \delta_{\mathrm{C}}\left(100.5 \mathrm{MHz}, \mathrm{CDCl}_{3}\right) 56.0\left(\mathrm{OCH}_{3}\right), 56.2$ $\left(\mathrm{OCH}_{3}\right), 114.1(\mathrm{CH}), 115.4\left(\mathrm{C}_{\text {quat }}\right), 118.3(\mathrm{CH}), 122.0(\mathrm{CH}), 131.6\left(\mathrm{C}_{\text {quat }}\right), 144.6(\mathrm{CH}), 148.5\left(\mathrm{C}_{\text {quat }}\right), 150.3$ $\left(\mathrm{C}_{\text {quat }}\right), 167.7\left(\mathrm{C}_{\text {quat }}, \mathrm{CO}\right)$. Found: $\mathrm{C}, 45.70 \%$; H, 3.86\%. Calcd. for $46.02 \% ; \mathrm{H}, 3.86 \%$.

(2E,4E)-5-Phenylpenta-2,4-dienoic acid (3p) (Procedure A). Pale yellow solid, mp. $165^{\circ} \mathrm{C}-166^{\circ} \mathrm{C}$ [Lit. mp. $\left.165^{\circ} \mathrm{C}[45]\right] ; v_{\max }\left(\mathrm{KBr} / \mathrm{cm}^{-1}\right) 3500-2850(\mathrm{bs}, \mathrm{OH}), 1678(\mathrm{C}=\mathrm{O}), 1609(\mathrm{C}=\mathrm{C}), 1582,1285 ; \delta_{\mathrm{H}}(400 \mathrm{MHz}$, $\left.\mathrm{CDCl}_{3}\right) 6.00\left(1 \mathrm{H}, \mathrm{d},{ }^{3} \mathrm{~J}=15.0 \mathrm{~Hz}\right), 6.90-6.96(2 \mathrm{H}, \mathrm{m}), 7.21-7.56(6 \mathrm{H}, \mathrm{m}) ; \delta_{\mathrm{C}}\left(100.5 \mathrm{MHz}, \mathrm{CDCl}_{3}\right) 120.1$, 126.1, 127.3, 128.9, 129.3, 135.9, 141.7, 146.9, 171.8; MS (EI, $70 \mathrm{eV}) \mathrm{m} / z(\%)=174(21)\left(\mathrm{M}^{+}\right), 129(100)$.

(E)-3-(Thien-2-yl)acrylic acid (3q) (Procedure A). Colorless solid, mp. $145^{\circ} \mathrm{C}$; [Lit. $145^{\circ} \mathrm{C}-148^{\circ} \mathrm{C}[41]$ ]. $v_{\max }\left(\mathrm{KBr} / \mathrm{cm}^{-1}\right) 3200-2500(\mathrm{bs}, \mathrm{OH}), 1677(\mathrm{C}=\mathrm{O}), 1615(\mathrm{C}=\mathrm{C}), 1410,1308,1274,1244,1218,1190,972,925$, $860,834,715,598,531 ; \delta_{\mathrm{H}}\left(400 \mathrm{MHz}, \mathrm{CDCl}_{3}\right) 6.24\left(1 \mathrm{H}, \mathrm{d},{ }^{3} \mathrm{~J}=15.6 \mathrm{~Hz}\right), 7.08\left(1 \mathrm{H}, \mathrm{dd},{ }^{3} J=5.2 \mathrm{~Hz},{ }^{3} J=4.0\right.$ $\mathrm{Hz}), 7.31\left(1 \mathrm{H}, \mathrm{d},{ }^{3} J=4.0 \mathrm{~Hz}\right), 7.43\left(1 \mathrm{H}, \mathrm{d},{ }^{3} J=5.2 \mathrm{~Hz}\right), 7.88\left(1 \mathrm{H}, \mathrm{d},{ }^{3} J=15.6 \mathrm{~Hz}\right) ; \delta_{\mathrm{C}}\left(100.5 \mathrm{MHz} \mathrm{CDCl}_{3}\right)$ $115.9(\mathrm{CH}), 128.2(\mathrm{CH}), 129.3(\mathrm{CH}), 131.7(\mathrm{CH}), 139.2\left(\mathrm{C}_{\text {quat }}\right), 139.4(\mathrm{CH}), 172.2\left(\mathrm{C}_{\text {quat }}, \mathrm{CO}\right)$; $\mathrm{MS}(\mathrm{FAB}$, 3-nitrobenzyl alcohol) $\mathrm{m} / \mathrm{z}(\%)=155(17)\left(\mathrm{MH}^{+}\right)$.

(E)-5-Bromothien-2-ylacrylic acid (3r) (Procedure A). Colorless solid, mp. $207^{\circ} \mathrm{C}$ [Lit. $209^{\circ} \mathrm{C}-210^{\circ} \mathrm{C}[46]$; 
$v_{\max }\left(\mathrm{KBr} / \mathrm{cm}^{-1}\right) 3430$ - $2597(\mathrm{bs}, \mathrm{OH}), 1688(\mathrm{C}=\mathrm{O}), 1615(\mathrm{C}=\mathrm{C}), 1433,1407,1298,1268,1211,966,795,533$; $\delta_{\mathrm{H}}\left(400 \mathrm{MHz}, \mathrm{DMSO}-\mathrm{d}^{6}\right) 6.11\left(1 \mathrm{H}, \mathrm{d},{ }^{3} J=16.0 \mathrm{~Hz}\right), 7.22\left(1 \mathrm{H}, \mathrm{d},{ }^{3} J=4.4 \mathrm{~Hz}\right), 7.29\left(1 \mathrm{H}, \mathrm{d},{ }^{3} J=4.4 \mathrm{~Hz}\right), 7.62$ $\left(1 \mathrm{H}, \mathrm{d},{ }^{3} J=16.0 \mathrm{~Hz}\right) ; \delta_{\mathrm{C}}\left(100.5 \mathrm{MHz}, \mathrm{DMSO}-\mathrm{d}^{6}\right) 115.4,118.6,132.3,132.9,136.3,141.1,167.6$.

(E)-3,4-Dimethoxycinnamic acid (3s) (Procedure A). Colorless solid, mp. $183^{\circ} \mathrm{C}$ [Lit. $181^{\circ} \mathrm{C}-183^{\circ} \mathrm{C}[41]$; $v_{\max }\left(\mathrm{KBr} / \mathrm{cm}^{-1}\right) 3300-2500(\mathrm{bs}, \mathrm{OH}), 1677(\mathrm{C}=\mathrm{O}), 1624,1596,1517,1463,1426,1340,1250,1140,1025$, $975,840,579,536 ; \delta_{\mathrm{H}}\left(400 \mathrm{MHz}, \mathrm{DMSO}-\mathrm{d}^{6}\right) 3.78\left(3 \mathrm{H}, \mathrm{s}, \mathrm{OCH}_{3}\right), 3.80\left(3 \mathrm{H}, \mathrm{s}, \mathrm{OCH}_{3}\right), 6.41\left(1 \mathrm{H}, \mathrm{d},{ }^{3} J=16.0\right.$ $\mathrm{Hz}), 6.86\left(1 \mathrm{H}, \mathrm{d},{ }^{3} \mathrm{~J}=8.0 \mathrm{~Hz}\right), 7.10(1 \mathrm{H}, \mathrm{s}), 7.13\left(1 \mathrm{H}, \mathrm{d},{ }^{3} J=8.0 \mathrm{~Hz}\right), 7.50\left(1 \mathrm{H}, \mathrm{d},{ }^{3} J=16.0 \mathrm{~Hz}\right), 12.19(1 \mathrm{H}, \mathrm{bs}$ $\mathrm{OH}) ; \delta_{\mathrm{C}}\left(100.5 \mathrm{MHz}, \mathrm{DMSO}-\mathrm{d}^{6}\right)$ 56.0, 56.1, 110.7, 112.0, 117.2, 123.1, 127.5, 144.6, 149.4, 151.2, 168.3; MS $(\mathrm{EI}, 70 \mathrm{eV}) m / z(\%)=208\left(\mathrm{M}^{+}, 100\right), 193\left(\mathrm{M}^{+}-\mathrm{CH}_{3}, 21\right)$.

(E)-3,4-Diethoxycinnamic acid (3t) (Procedure A). Colorless solid, mp. $154^{\circ} \mathrm{C}$ [Lit. $\left.156^{\circ} \mathrm{C}[47]\right] \delta_{\mathrm{H}}(400$ $\left.\mathrm{MHz}, \mathrm{CDCl}_{3}\right) 1.46\left(6 \mathrm{H}, \mathrm{t},{ }^{3} \mathrm{~J}=6.8 \mathrm{~Hz}, 2 \mathrm{CH}_{3}\right), 4.12\left(2 \mathrm{H}, \mathrm{q},{ }^{3} \mathrm{~J}=6.8 \mathrm{~Hz}, \mathrm{OCH}_{2}\right), 4.13\left(2 \mathrm{H}, \mathrm{q},{ }^{3} \mathrm{~J}=6.8 \mathrm{~Hz}\right.$, $\left.\mathrm{OCH}_{2}\right), 6.28\left(1 \mathrm{H}, \mathrm{d},{ }^{3} J=16.0 \mathrm{~Hz}\right), 6.85\left(1 \mathrm{H}, \mathrm{d},{ }^{3} \mathrm{~J}=8.0 \mathrm{~Hz}\right), 7.07(1 \mathrm{H}, \mathrm{s}), 7.08\left(1 \mathrm{H}, \mathrm{d},{ }^{3} J=8.0 \mathrm{~Hz}\right), 7.70(1 \mathrm{H}, \mathrm{d}$, $\left.{ }^{3} J=16.0 \mathrm{~Hz}\right) ; \delta_{\mathrm{C}}\left(100.5 \mathrm{MHz}, \mathrm{CDCl}_{3}\right) 14.6,14.7,64.5,64.6,111.9,112.6,114.6,123.1,126.9,147.1,148.8$, $151.3,172.4$.

(E)-3,4-(Methylenedioxy)-cinnamic acid (3u) (Procedure A). Mp. 214 ${ }^{\circ} \mathrm{C}$ [Lit. $\left.242^{\circ} \mathrm{C}-244^{\circ} \mathrm{C}[41]\right] ; v$ $\left(\mathrm{KBr} / \mathrm{cm}^{-1}\right) 3200-2500(\mathrm{bs}, \mathrm{OH}), 1693(\mathrm{C}=\mathrm{O}), 1624(\mathrm{C}=\mathrm{C}), 1495,1446,1314,1251,1100,1036,924,805 ; \delta_{\mathrm{H}}$ $\left(400 \mathrm{MHz}, \mathrm{DMSO}-\mathrm{d}^{6}\right) 6.08(2 \mathrm{H}, \mathrm{s}), 6.39\left(1 \mathrm{H}, \mathrm{d},{ }^{3} J=15.6 \mathrm{~Hz}\right), 6.95\left(1 \mathrm{H}, \mathrm{d},{ }^{3} J=8.0 \mathrm{~Hz}\right), 7.16\left(1 \mathrm{H}, \mathrm{dd},{ }^{3} J 8.0\right.$ $\left.\mathrm{Hz},{ }^{4} J=1.6 \mathrm{~Hz}\right), 7.37\left(1 \mathrm{H}, \mathrm{d},{ }^{4} J=1.6 \mathrm{~Hz}\right), 7.51\left(1 \mathrm{H}, \mathrm{d},{ }^{3} J=15.6 \mathrm{~Hz}\right) ; \delta_{\mathrm{C}}\left(100.5 \mathrm{MHz}, \mathrm{DMSO}-\mathrm{d}^{6}\right) 101.4,106.6$, 108.4, 117.0, 124.5, 128.6, 143.7, 147.9, 149.0, 167.7; MS (EI, $70 \mathrm{eV}) \mathrm{m} / z(\%)=192\left(\mathrm{M}^{+}, 100\right), 145(13)$.

(E)-2-Chlorocinnamic acid (3v) (Procedure A). Colorless solid; mp. $200^{\circ} \mathrm{C}$ [Lit. mp. $208^{\circ} \mathrm{C}-210^{\circ} \mathrm{C}[41]$; $v_{\max }\left(\mathrm{KBr} / \mathrm{cm}^{-1}\right) 3050-2500(\mathrm{bs}, \mathrm{OH}), 2971(\mathrm{C}-\mathrm{H}), 1687(\mathrm{C}=\mathrm{O}), 1622(\mathrm{C}=\mathrm{C}), 1427,1281,1224,1041,978$, 921, 727, 595, 560; for $\boldsymbol{E}-3 \mathrm{v}: \delta_{\mathrm{H}}\left(400 \mathrm{MHz}, \mathrm{DMSO}-\mathrm{d}^{6}\right) 6.57\left(1 \mathrm{H}, \mathrm{d},{ }^{3} J=16.0 \mathrm{~Hz}\right), 7.51\left(1 \mathrm{H}, \mathrm{dd},{ }^{3} J=7.6 \mathrm{~Hz},{ }^{4} J\right.$ $=1.6 \mathrm{~Hz}), 7.35-7.45(2 \mathrm{H}, \mathrm{m}), 7.84\left(1 \mathrm{H}, \mathrm{d},{ }^{3} J=16.0 \mathrm{~Hz}\right), 7.89\left(1 \mathrm{H}, \mathrm{dd},{ }^{3} J=7.6 \mathrm{~Hz},{ }^{4} J=2.0 \mathrm{~Hz}\right) ; \delta_{\mathrm{C}}(100.5$ MHz, DMSO-d $\left.{ }^{6}\right)$ 122.7, 128.3, 128.6, 130.4, $132.2(2 \mathrm{C}), 134.0,139.2,167.7\left(\mathrm{C}_{\text {quat, }}, \mathrm{CO}\right)$; for $Z \mathbf{Z}-3 \mathbf{v}: \delta_{\mathrm{H}}(400$ MHz, DMSO-d $\left.{ }^{6}\right) 6.10\left(1 \mathrm{H}, \mathrm{d},{ }^{3} J=12.0 \mathrm{~Hz}\right), 7.03\left(1 \mathrm{H}, \mathrm{d},{ }^{3} J=12.0 \mathrm{~Hz}\right), 7.26-7.45(4 \mathrm{H}, \mathrm{m}) ; \delta_{\mathrm{C}}(100.5 \mathrm{MHz}$ DMSO- $\left.\mathrm{d}^{6}\right) 124.2,127.1,129.3,131.1,132.3(2 \mathrm{C}), 134.5,138.5,167.2\left(\mathrm{C}_{\text {quat }}, \mathrm{CO}\right)$; MS $(\mathrm{EI}, 70 \mathrm{eV}) \mathrm{m} / \mathrm{z}(\%)=$ $182(15)\left(\left[{ }^{35} \mathrm{Cl}\right] \mathrm{M}^{+}\right), 147(100)$.

Ethyl (E)-4-nitrocinnamate $(3 w)$ and $(E)$-4-nitrocinnamic acid $(3 x)$. A mixture of 4-nitrobenzaldehyde $(1 \mathbf{w}, 1.51 \mathrm{~g}, 10 \mathrm{mmol})$ and phosphorane $2 \mathbf{a}(4.87 \mathrm{~g}, 14 \mathrm{mmol})$ in chloroform $(0.5 \mathrm{~mL})$ was heated to $120^{\circ} \mathrm{C}$ for $45 \mathrm{~min}$. The cooled solution is subjected directly to column chromatography on silica gel $\left(\mathrm{CHCl}_{3} / \mathrm{ether} / \mathrm{hexane}\right.$ $1: 1: 1)$ to give $3 \mathbf{w}(1.97 \mathrm{~g}, 89 \%)$ as a light yellow solid; mp. $140^{\circ} \mathrm{C}$; [Lit. $138^{\circ} \mathrm{C}-140^{\circ} \mathrm{C}[41]$ ]; $v_{\max }\left(\mathrm{KBr} / \mathrm{cm}^{-1}\right)$ 3106, $3079\left(\mathrm{C}-\mathrm{H}_{\text {arom. }}\right), 2983\left(\mathrm{C}-\mathrm{H}_{\text {aliph }}\right), 2939\left(\mathrm{C}-\mathrm{H}_{\text {aliph }}\right), 1712(\mathrm{C}=\mathrm{O}), 1645,1594,1340\left(\mathrm{NO}_{2(\mathrm{~s})}\right), 1189,1177$, $1110,1029,1189,1171,1110,1028,978,845,759 ; \delta_{\mathrm{H}}\left(400 \mathrm{MHz}, \mathrm{CDCl}_{3}\right) 1.35\left(3 \mathrm{H}, \mathrm{t},{ }^{3} J=7.2 \mathrm{~Hz}\right), 4.29(2 \mathrm{H}, \mathrm{q}$, $\left.{ }^{3} J=7.2 \mathrm{~Hz}\right), 6.55\left(1 \mathrm{H}, \mathrm{d},{ }^{3} J=16.4 \mathrm{~Hz}\right), 7.70\left(1 \mathrm{H}, \mathrm{d},{ }^{3} J=16.4 \mathrm{~Hz}\right), 7.66\left(2 \mathrm{H}, \mathrm{d},{ }^{3} J=8.8 \mathrm{~Hz}\right), 8.25\left(2 \mathrm{H}, \mathrm{d},{ }^{3} J=\right.$ $8.8 \mathrm{~Hz})$. A solution of $\mathbf{3 w}(1.95 \mathrm{~g}, 8.82 \mathrm{mmol})$ in a mixture of aq. $\mathrm{NaOH}(10 \mathrm{w} \%, 50 \mathrm{~mL})$ and methanol $(10 \mathrm{~mL})$ was heated at $65^{\circ} \mathrm{C}$ for $12 \mathrm{~h}$. Thereafter, the solution was filtered, and the filtrate was acidified with half-conc. aq. $\mathrm{HCl}$. The formed precipitate was filtered, washed with water $(3 \times 20 \mathrm{~mL})$ and air-dried to give $3 \mathbf{x}(1.62 \mathrm{~g}$, $95 \%$ ) as a light yellowish solid; mp. $287^{\circ} \mathrm{C}$ [Lit. mp. $289^{\circ} \mathrm{C}[40]$ ]; $v_{\max }\left(\mathrm{KBr} / \mathrm{cm}^{-1}\right) 3500-2499$ (bs, $\left.\mathrm{OH}\right), 1686$ $(\mathrm{C}=\mathrm{O}), 1628(\mathrm{C}=\mathrm{C}), 1522\left(\mathrm{NO}_{2(\mathrm{as})}\right), 1426,1350\left(\mathrm{NO}_{2(\mathrm{~s})}\right), 1308,1283,1222,985,836,716 ; \delta_{\mathrm{H}}(400 \mathrm{MHz}$ DMSO-d $\left.^{6}\right) 6.69\left(1 \mathrm{H}, \mathrm{d},{ }^{3} \mathrm{~J}=16.0 \mathrm{~Hz}\right), 7.64\left(1 \mathrm{H}, \mathrm{d},{ }^{3} \mathrm{~J}=16.0 \mathrm{~Hz}\right), 7.92\left(2 \mathrm{H}, \mathrm{d},{ }^{3} \mathrm{~J}=8.8 \mathrm{~Hz}\right), 8.20\left(2 \mathrm{H}, \mathrm{d},{ }^{3} \mathrm{~J}=8.8\right.$ $\mathrm{Hz}) ; \delta_{\mathrm{C}}\left(100.5 \mathrm{MHz}, \mathrm{DMSO}-\mathrm{d}^{6}\right) 124.4(2 \mathrm{C}, \mathrm{CH}), 129.7(2 \mathrm{C}, \mathrm{CH}), 123.9(\mathrm{CH}), 141.1\left(\mathrm{C}_{\text {quat }}\right), 141.8(\mathrm{CH}), 148.4$ $\left(\mathrm{C}_{\text {quat }}\right), 167.5\left(\mathrm{C}_{\text {quat }}, \mathrm{CO}\right)$; MS (EI, $\left.70 \mathrm{eV}\right) \mathrm{m} / z(\%) 193\left(\mathrm{M}^{+}, 65\right), 176(18), 147$ (15).

(Z)-4-Benzyloxycinnamic acid (3y) (Procedure A). Colorless solid; mp. $168^{\circ} \mathrm{C} ; \delta_{\mathrm{H}}\left(400 \mathrm{MHz}, \mathrm{DMSO}^{6} \mathrm{~d}^{6}\right)$ $5.11\left(2 \mathrm{H}, \mathrm{s}, \mathrm{OCH}_{2}\right), 5.79\left(1 \mathrm{H}, \mathrm{d},{ }^{3} J=12.8 \mathrm{~Hz}\right), 6.80\left(1 \mathrm{H}, \mathrm{d},{ }^{3} \mathrm{~J}=12.8 \mathrm{~Hz}\right), 6.98\left(2 \mathrm{H}, \mathrm{d},{ }^{3} J=8.8 \mathrm{~Hz}\right), 7.40(2 \mathrm{H}, \mathrm{d}$, $3 J=8.4 \mathrm{~Hz}), 7.57\left(2 \mathrm{H}, \mathrm{d},{ }^{3} J=8.4 \mathrm{~Hz}\right), 7.67\left(2 \mathrm{H}, \mathrm{d},{ }^{3} J=8.8 \mathrm{~Hz}\right) ; \delta_{\mathrm{C}}\left(100.5 \mathrm{MHz}, \mathrm{DMSO}-\mathrm{d}^{6}\right) 68.8\left(\mathrm{OCH}_{2}\right)$, $114.7(2 \mathrm{C}, \mathrm{CH}), 118.9,121.4,128.0,129.2,130.3(2 \mathrm{C}, \mathrm{CH}), 131.8(2 \mathrm{C}, \mathrm{CH}), 132.4(2 \mathrm{C}, \mathrm{CH}), 136.8,141.2$, $159.2\left(\mathrm{C}_{\text {quat }}\right), 168.0\left(\mathrm{C}_{\text {quat }}, \mathrm{CO}\right)$; $\mathrm{MS}$ (Ion trap): $m / z 255\left(\mathrm{MH}^{+}\right)$.

Ethyl 3(E)-(9-anthranyl)propenoate (3z-Et). A mixture of 9-anthranylcarbaldehyde (1y, $1.0 \mathrm{~g}, 4.85 \mathrm{mmol})$ and ethoxycarbonylmethylidenephosphorane $(\mathbf{2 a}, 2.36 \mathrm{~g}, 6.79 \mathrm{mmol})$ is heated at $130^{\circ} \mathrm{C}$ for $3 \mathrm{~h}$. Thereafter, an additional amount of $2 \mathbf{a}(348 \mathrm{mg}, 1.0 \mathrm{mmol})$ is added and the reaction mixture heated for another hour at $135^{\circ} \mathrm{C}$. The cooled solution is subjected directly to column chromatography on silica gel (eluent: $\mathrm{M}^{t} \mathrm{BE} / \mathrm{CHCl}_{3} / \mathrm{hexane}$ $1: 1: 7)$ to give 3z-Et as a yellow-orange solid $(1.23 \mathrm{~g}, 92 \%)$, mp. $80^{\circ} \mathrm{C}$ [Lit. $80^{\circ} \mathrm{C}$ [48]]; $\delta_{\mathrm{H}}\left(400 \mathrm{MHz} \mathrm{CDCl}_{3}\right)$ $1.35\left(3 \mathrm{H}, \mathrm{t},{ }^{3} J=7.2 \mathrm{~Hz}\right), 4.31\left(2 \mathrm{H}, \mathrm{q},{ }^{3} J=7.2 \mathrm{~Hz}, \mathrm{OCH}_{2}\right), 6.36\left(1 \mathrm{H}, \mathrm{d},{ }^{3} J=16.0 \mathrm{~Hz}\right), 7.43(4 \mathrm{H}, \mathrm{m}), 7.95(2 \mathrm{H}$, 
m), $8.17(2 \mathrm{H}, \mathrm{m}), 8.39(1 \mathrm{H}, \mathrm{s}), 8.57\left(1 \mathrm{H}, \mathrm{d},{ }^{3} \mathrm{~J}=16.0 \mathrm{~Hz}\right) ; \delta_{\mathrm{C}}\left(100.5 \mathrm{MHz}, \mathrm{CDCl}_{3}\right) 14.5,61.0,125.2,125.4$, 127.2, 128.2, 128.8, 129.3, 129.4, 131.2, 141.9, 166.5; MS: Found: $299.1040\left(\mathrm{C}_{19} \mathrm{H}_{16} \mathrm{O}_{2}+\mathrm{Na}\right)^{+}$; Calcd. for $\mathrm{C}_{19} \mathrm{H}_{16} \mathrm{O}_{2} \mathrm{Na}: 299.1048$.

Alternatively, to the reaction mixture of $\mathbf{1 y}$ and $\mathbf{2 a}$, aq. $\mathrm{NaOH}(10 \mathrm{w} \%, 15 \mathrm{~mL})$ was added after $4 \mathrm{~h}$, and the resulting solution was kept at reflux for $14 \mathrm{~h}$. Thereafter, the solution was cooled and the precipitate was filtered off. The precipitate which contains both triphenylphosphine oxide and sodium 3(E)-(9-anthranyl)propenoate (3z-Na, see below) was washed diligently with hot water, to dissolve the remainder of the salt. Acidification of the cool filtrate with half conc. aq. $\mathrm{HCl}$ provides a precipitate, which was filtered, washed with water and dried to yield 3-(9-anthranyl)propenoic acid (3z) $(1.02 \mathrm{~g}, 85 \%)$ as a yellow solid; mp. $242^{\circ} \mathrm{C}$ [Lit. mp. $245^{\circ} \mathrm{C}$ [49]]; $v_{\max }\left(\mathrm{KBr} / \mathrm{cm}^{-1}\right) 3500-2495(\mathrm{bs}, \mathrm{OH}), 3424,3051\left(\mathrm{C}-\mathrm{H}_{\text {arom. }}\right), 3003\left(\mathrm{C}-\mathrm{H}_{\text {arom. }}\right), 2898\left(\mathrm{C}-\mathrm{H}_{\text {aliph }}\right), 1690(\mathrm{C}=\mathrm{O})$, $1630,1425,1307,1200,725 ; \delta_{\mathrm{H}}\left(400 \mathrm{MHz}, \mathrm{DMSO}-\mathrm{d}^{6}\right) 6.32\left(1 \mathrm{H}, \mathrm{d},{ }^{3} \mathrm{~J}=16.4 \mathrm{~Hz}\right), 7.57(4 \mathrm{H}, \mathrm{m}), 8.12(4 \mathrm{H}, \mathrm{m})$, $8.46\left(1 \mathrm{H}, \mathrm{d},{ }^{3} J=16.4 \mathrm{~Hz}\right), 8.64(1 \mathrm{H}, \mathrm{s}), 12.75(\mathrm{bs}, \mathrm{OH}) ; \delta_{\mathrm{C}}\left(100.5 \mathrm{MHz}, \mathrm{DMSO}-\mathrm{d}^{6}\right) 125.3(\mathrm{CH}), 126.0(\mathrm{CH})$, $127.2(\mathrm{CH}), 128.4\left(\mathrm{C}_{\text {quat }}\right), 128.8\left(\mathrm{C}_{\text {quat }}\right), 129.1(\mathrm{CH}), 129.3(\mathrm{CH}), 129.5\left(\mathrm{C}_{\text {quat }}\right), 131.2(\mathrm{CH}), 141.0(\mathrm{CH}), 157.3$ $\left(\mathrm{C}_{\text {quat }}, \mathrm{CO}\right)$.

When heated with $10 \mathrm{w} \%$ aq. $\mathrm{NaOH}, 3-(9$-anthranyl)propenoic acid (3z) gives sodium 3(E)-(9-anthranyl) propenoate (3z-Na) as golden, shiny leaflets $v_{\max }\left(\mathrm{KBr} / \mathrm{cm}^{-1}\right) 3620-2850(\mathrm{bs}, \mathrm{OH}), 3611(\mathrm{v}), 1635,1540(\mathrm{~s})$, $1441,1392,1285,991,881,734 ; \delta_{\mathrm{H}}\left(400 \mathrm{MHz}, \mathrm{CDCl}_{3}\right) 6.14\left(1 \mathrm{H}, \mathrm{d},{ }^{3} J=16.0 \mathrm{~Hz}\right), 7.50(4 \mathrm{H}, \mathrm{m}), 7.90\left(1 \mathrm{H}, \mathrm{d},{ }^{3} J\right.$ $=16.0 \mathrm{~Hz}), 8.07(2 \mathrm{H}, \mathrm{m}), 8.20(2 \mathrm{H}, \mathrm{m}), 8.50(1 \mathrm{H}, \mathrm{s})$.

4-Nitrobenzoic acid (4) and 4-nitrobenzyl alcohol (5). 4-Nitrobenzaldehyde (1w, $1.51 \mathrm{~g}, 10 \mathrm{mmol})$ and phosphorane $2 \mathrm{a}(4.87 \mathrm{~g}, 14 \mathrm{mmol})$ was given to an aq. $\mathrm{NaOH}$ solution $(9.1 \mathrm{w} \%, 3.5 \mathrm{~g}(87.5 \mathrm{mmol}) \mathrm{NaOH}$ in 35 $\mathrm{mL} \mathrm{H}_{2} \mathrm{O}$ ) and the resulting suspension was stirred at $75^{\circ} \mathrm{C}$ for $20 \mathrm{~min}$. With the addition of 4-nitrobenzaldehyde, the solution turned dark-red. The solution was filtered, and the filtrate was extracted with dichloromethane $(3 \times$ $25 \mathrm{~mL}$ ). Then, the aqueous solution was acidified with half-conc. aq. $\mathrm{HCl}$ to give 4-nitrobenzoic acid (4) (800 $\mathrm{mg}, 48 \%)$ as a colorless solid, mp. $237^{\circ} \mathrm{C}$ [Lit. $\left.237^{\circ} \mathrm{C}-240^{\circ} \mathrm{C}[41]\right] ; v\left(\mathrm{KBr} / \mathrm{cm}^{-1}\right) 3065(\mathrm{OH}), 2672,1695$ $(\mathrm{C}=\mathrm{O}), 1609(\mathrm{C}=\mathrm{C}), 1543,1433 ; \delta_{\mathrm{H}}\left(400 \mathrm{MHz}, \mathrm{CDCl}_{3}\right) 8.13\left(2 \mathrm{H}, \mathrm{d},{ }^{3} J=9.2 \mathrm{~Hz}\right), 8.28\left(2 \mathrm{H}, \mathrm{d},{ }^{3} J=9.2 \mathrm{~Hz}\right) ; \delta_{\mathrm{C}}$ $\left(100.5 \mathrm{MHz}, \mathrm{DMSO}-\mathrm{d}^{6}\right) 123.5(2 \mathrm{C}, \mathrm{CH}), 131.1(2 \mathrm{C}, \mathrm{CH}), 136.4\left(\mathrm{C}_{\text {quat }}\right), 150.5\left(\mathrm{C}_{\text {quat }}\right), 166.8\left(\mathrm{C}_{\text {quat }}, \mathrm{CO}\right)$. Thereafter the filter cake of the first filtration was taken up in $\mathrm{CHCl}_{3}(50 \mathrm{~mL})$ and combined with the $\mathrm{CH}_{2} \mathrm{Cl}_{2}$ extract. The combined extract was dried over anhydrous $\mathrm{MgSO}_{4}$ and concentrated in vacuo. The residue was subjected to column chromatography on silica gel $\left(\mathrm{CHCl}_{3}\right.$-hexane-ether 1:1:1) to give 4-nitrobenzyl alcohol $(\mathbf{5}, 540$ $\mathrm{mg}, 35 \%)$ as a colorless solid, $\mathrm{mp} .93^{\circ} \mathrm{C}\left[\right.$ Lit. $\left.92^{\circ} \mathrm{C}-94^{\circ} \mathrm{C}[48]\right] ; v\left(\mathrm{KBr} / \mathrm{cm}^{-1}\right) 3550(\mathrm{bs}, \mathrm{OH}), 2954(\mathrm{C}-\mathrm{H}), 2925$ $(\mathrm{C}-\mathrm{H}), 1508\left(\mathrm{NO}_{2(\mathrm{a})}\right), 1458\left(\mathrm{NO}_{2(\mathrm{~s})}\right) ; \delta_{\mathrm{H}}\left(400 \mathrm{MHz}, \mathrm{CDCl}_{3}\right) 2.11\left(1 \mathrm{H}, \mathrm{t},{ }^{3} J=5.6 \mathrm{~Hz}, \mathrm{OH}\right), 4.83\left(2 \mathrm{H}, \mathrm{d},{ }^{3} J=5.6\right.$ $\mathrm{Hz}), 7.52\left(2 \mathrm{H}, \mathrm{cd},{ }^{3} J=8.8 \mathrm{~Hz}\right), 8.21\left(2 \mathrm{H}, \mathrm{cd},{ }^{3} J=8.8 \mathrm{~Hz}\right) ; \delta_{\mathrm{H}}\left(400 \mathrm{MHz}, \mathrm{CDCl}_{3}\right) 64.0\left(\mathrm{CH}_{2}\right), 123.7(2 \mathrm{C}, \mathrm{CH})$, $127.1(2 \mathrm{C}, \mathrm{CH}), 147.3\left(\mathrm{C}_{\text {quat }}\right), 148.4\left(\mathrm{C}_{\text {quat }}\right)$; MS (EI, $\left.70 \mathrm{eV}\right) \mathrm{m} / \mathrm{z}(\%) 153\left(55, \mathrm{M}^{+}\right), 77(100)$.

(E)-4-Hydroxycinnamic acid (6) [50]. A mixture of $\mathbf{1 z}(835 \mathrm{mg}, 6.84 \mathrm{mmol})$ and $\mathbf{2 a}(3.35 \mathrm{~g}, 9.63 \mathrm{mmol})$ was heated at $135^{\circ} \mathrm{C}$ for $1 \mathrm{~h}$. Thereafter, aq. $\mathrm{NaOH}(10 \mathrm{w} \%, 10 \mathrm{~mL})$ was added to the cooled mixture, and it was stirred for $12 \mathrm{~h}$ at $\mathrm{rt}$. The precipitated triphenylphosphine oxide was filtered off, and the filter-cake was washed with water $(2 \times 15 \mathrm{~mL})$. The filtrate, combined with the washings, was acidified with half-conc. aq. HCl. The formed precipitate was filtered, washed with water $(2 \times 15 \mathrm{~mL})$ and air-dried to yield $6(850 \mathrm{mg}, 74 \%)$ as a colorless solid, mp. $211^{\circ} \mathrm{C}-212^{\circ} \mathrm{C}$; IR $\left(\mathrm{KBr} / \mathrm{cm}^{-1}\right) v_{\max } 3379$ (bs, OH), $1672(\mathrm{C}=\mathrm{O}), 1628,1602(\mathrm{C}=\mathrm{C}), 1512$, 1449, 1313, 1244 (C-O), 1214 (C-O), 1172, 978, 832, 690; $\delta_{\mathrm{H}}\left(400 \mathrm{MHz}, \mathrm{DMSO}-\mathrm{d}^{6}\right) 6.23\left(1 \mathrm{H}, \mathrm{d},{ }^{3} J=16.0 \mathrm{~Hz}\right)$, $6.76\left(2 \mathrm{H}, \mathrm{d},{ }^{3} J=8.8 \mathrm{~Hz}\right), 7.19\left(1 \mathrm{H}, \mathrm{d},{ }^{3} J=16.0 \mathrm{~Hz}\right), 7.34\left(2 \mathrm{H}, \mathrm{d},{ }^{3} J=8.8 \mathrm{~Hz}\right) ; \delta_{\mathrm{H}}\left(100.5 \mathrm{MHz}, \mathrm{DMSO}-\mathrm{d}^{6}\right) 115.0$ $(\mathrm{CH}), 115.9(2 \mathrm{C}, \mathrm{CH}), 127.2\left(\mathrm{C}_{\text {quat }}\right), 129.3(2 \mathrm{C}, \mathrm{CH}), 138.9(\mathrm{CH}), 158.7\left(\mathrm{C}_{\text {quat }}\right), 171.0\left(\mathrm{C}_{\text {quat }}, \mathrm{CO}\right)$.

While general procedure A was used for the preparation of $\mathbf{8 b}, \mathbf{8 d} / \mathbf{8 e}$, and $8 \mathbf{8}$, the $E$ - and $Z$-isomers of $\mathbf{8 b}$ and 8f and compound 8d and 8e were separated by column chromatography on silica gel $\left(\mathrm{CHCl}_{3} /\right.$ ether 10:1) and crystallized from a mixture of $\mathrm{CHCl}_{3}$-hexane (1:4).

(E)-3-Phenylbut-2-enoic acid (8a) (Procedure A). Colorless solid, mp. $129^{\circ} \mathrm{C}-131^{\circ} \mathrm{C}$ [Lit. $\left.131^{\circ} \mathrm{C}[51]\right] ; \delta_{\mathrm{H}}$ $\left(400 \mathrm{MHz}, \mathrm{DMSO}-\mathrm{d}^{6}\right) 2.49\left(3 \mathrm{H}, \mathrm{d},{ }^{4} J=1.6 \mathrm{~Hz}\right), 6.10\left(1 \mathrm{H}, \mathrm{q},{ }^{4} J=1.6 \mathrm{~Hz}\right), 7.40-7.42(3 \mathrm{H}, \mathrm{m}), 7.55-7.57(2 \mathrm{H}$, m), $12.27(1 \mathrm{H}, \mathrm{bs}, \mathrm{OH}) ; \delta_{\mathrm{C}}\left(100.5 \mathrm{MHz}, \mathrm{DMSO}-\mathrm{d}^{6}\right) 17.7\left(\mathrm{CH}_{3}\right), 117.9(\mathrm{CH}), 126.6(2 \mathrm{C}, \mathrm{CH}), 129.1(2 \mathrm{C}, \mathrm{CH})$, $129.5(\mathrm{CH}), 141.9\left(\mathrm{C}_{\text {quat }}\right), 154.2\left(\mathrm{C}_{\text {quat }}\right), 168.0\left(\mathrm{C}_{\text {quat, }}, \mathrm{CO}\right)$.

(Z)-3-(4-Chlorophenyl)but-2-enoic acid $(\boldsymbol{Z}-8 b)[52]$ (Procedure A). $v\left(\mathrm{KBr} / \mathrm{cm}^{-1}\right) 3150-2550$ (bs, OH), $1678(\mathrm{C}=\mathrm{O}), 1617(\mathrm{C}=\mathrm{C}), 1432,1289,1210,1073,819,713 ; \delta_{\mathrm{H}}\left(400 \mathrm{MHz}, \mathrm{DMSO}-\mathrm{d}^{6}\right) 2.11\left(3 \mathrm{H}, \mathrm{d},{ }^{4} J=1.6 \mathrm{~Hz}\right.$, $\left.\mathrm{CH}_{3}\right), 5.92\left(1 \mathrm{H}, \mathrm{q},{ }^{4} J=1.6 \mathrm{~Hz}\right), 7.25\left(2 \mathrm{H}, \mathrm{d},{ }^{3} J=8.8 \mathrm{~Hz}\right), 7.39\left(2 \mathrm{H}, \mathrm{d},{ }^{3} J=8.8 \mathrm{~Hz}\right) ; \delta_{\mathrm{H}}\left(400 \mathrm{MHz}, \mathrm{DMSO}-\mathrm{d}^{6}\right)$ $26.7\left(\mathrm{CH}_{3}\right), 119.4(\mathrm{CH}), 128.2(\mathrm{CH}, 2 \mathrm{C}), 129.4(\mathrm{CH}, 2 \mathrm{C}), 132.6,139.9,152.6,166.8\left(\mathrm{C}_{\text {quat }}, \mathrm{CO}\right) .(\boldsymbol{E})-3-(4-$ 
Chlorophenyl)but-2-enoic acid $(\boldsymbol{E}-8 \mathrm{~b})$. Beige solid, mp. $133^{\circ} \mathrm{C}\left[\right.$ Lit. $\left.135^{\circ} \mathrm{C}\right] ; \delta_{\mathrm{H}}\left(400 \mathrm{MHz}, \mathrm{DMSO}-\mathrm{d}^{6}\right) 2.47$ $\left(3 \mathrm{H}, \mathrm{d},{ }^{4} \mathrm{~J}=1.6 \mathrm{~Hz}, \mathrm{CH}_{3}\right), 6.12\left(1 \mathrm{H}, \mathrm{q},{ }^{4} J=1.6 \mathrm{~Hz}\right), 7.47\left(2 \mathrm{H}, \mathrm{d},{ }^{3} J=8.8 \mathrm{~Hz}\right), 7.59\left(2 \mathrm{H}, \mathrm{d},{ }^{3} J=8.8 \mathrm{~Hz}\right), 12.2$ $(1 \mathrm{H}, \mathrm{bs}, \mathrm{OH}) ; \delta_{\mathrm{C}}\left(100.5 \mathrm{MHz}, \mathrm{DMSO}-\mathrm{d}^{6}\right) 17.5\left(\mathrm{CH}_{3}\right), 118.6(\mathrm{CH}), 128.5(\mathrm{CH}, 2 \mathrm{C}), 129.0(\mathrm{CH}, 2 \mathrm{C}), 134.2$, $140.7,152.6,167.8\left(\mathrm{CO}, \mathrm{C}_{\text {quat }}\right)$.

(E)-3-(4-Bromophenyl)but-2-enoic acid (8c) (Procedure A). Colorless solid, mp. $116^{\circ} \mathrm{C}$ [Lit. $\left.116^{\circ} \mathrm{C}[11]\right] ; v$ $\left(\mathrm{KBr} / \mathrm{cm}^{-1}\right) 3150-2550(\mathrm{bs}, \mathrm{OH}), 1681(\mathrm{C}=\mathrm{O}), 1610(\mathrm{C}=\mathrm{C}), 817 ; \delta_{\mathrm{H}}\left(400 \mathrm{MHz}, \mathrm{DMSO}-\mathrm{d}^{6}\right) 2.47\left(3 \mathrm{H}, \mathrm{q},{ }^{4} J=\right.$ $\left.1.2 \mathrm{~Hz}, \mathrm{CH}_{3}\right), 6.12\left(1 \mathrm{H}, \mathrm{d},{ }^{4} J=1.2 \mathrm{~Hz}\right), 7.51\left(2 \mathrm{H}, \mathrm{d},{ }^{3} J=8.4 \mathrm{~Hz}\right), 7.60\left(2 \mathrm{H}, \mathrm{d},{ }^{3} J=8.4 \mathrm{~Hz}\right), 12.30(1 \mathrm{H}, \mathrm{bs}, \mathrm{OH})$; $\delta_{\mathrm{C}}\left(100.5 \mathrm{MHz}, \mathrm{DMSO}-\mathrm{d}^{6}\right) 17.5,118.6,122.9,128.8(2 \mathrm{C}), 131.9(2 \mathrm{C}), 141.0,152.7,167.8$.

(E)-3-(4-Iodophenyl)but-2-enoic acid (8d) (Procedure A) [53]. Colorless solid; mp. $161^{\circ} \mathrm{C} ; v\left(\mathrm{KBr} / \mathrm{cm}^{-1}\right)$ 3150 - 2550 (bs, OH), $1683(\mathrm{C}=\mathrm{O}), 1608(\mathrm{C}=\mathrm{C}), 1436,1278(\mathrm{C}-\mathrm{O}), 1208(\mathrm{C}-\mathrm{O}), 994,814,708 ; \delta_{\mathrm{H}}(400 \mathrm{MHz}$, DMSO-d $\left.{ }^{6}\right) 2.45\left(3 \mathrm{H}, \mathrm{q},{ }^{4} \mathrm{~J}=1.2 \mathrm{~Hz}, \mathrm{CH}_{3}\right), 6.11\left(1 \mathrm{H}, \mathrm{q},{ }^{4} \mathrm{~J}=1.2 \mathrm{~Hz}\right), \delta_{\mathrm{H}}\left(400 \mathrm{MHz}, \mathrm{CDCl}_{3}\right) 2.58\left(3 \mathrm{H}, \mathrm{q},{ }^{4} \mathrm{~J}=1.2\right.$ $\left.\mathrm{Hz}, \mathrm{CH}_{3}\right), 6.15\left(1 \mathrm{H}, \mathrm{q},{ }^{4} J=1.2 \mathrm{~Hz}\right), 7.36\left(2 \mathrm{H}, \mathrm{d},{ }^{3} J=8.4 \mathrm{~Hz}\right), 7.43\left(2 \mathrm{H}, \mathrm{d},{ }^{3} J=8.4 \mathrm{~Hz}\right) ; \delta_{\mathrm{C}}(100.5 \mathrm{MHz}$, DMSO-d $\left.{ }^{6}\right) 17.4,96.1,118.4,128.8(2 \mathrm{C}), 137.8(2 \mathrm{C}), 141.4,152.9,167.8 ; \delta_{\mathrm{C}}\left(100.5 \mathrm{MHz}, \mathrm{CDCl}_{3}\right) 18.2\left(\mathrm{CH}_{3}\right)$, $116.5(\mathrm{CH}), 127.7(\mathrm{CH}, 2 \mathrm{C}), 128.9(\mathrm{CH}, 2 \mathrm{C}), 135.4\left(\mathrm{C}_{\text {quat }}\right), 140.3\left(\mathrm{C}_{\text {quat }}\right), 157.1\left(\mathrm{C}_{\text {quat }}\right), 171.6\left(\mathrm{C}_{\text {quat }}, \mathrm{CO}\right)$, and 3-(4-iodophenyl)but-3-enoic acid $(\mathbf{8 e})$ as a colorless solid, $\mathrm{mp} .143^{\circ} \mathrm{C} ; \mathrm{v}\left(\mathrm{KBr} / \mathrm{cm}^{-1}\right) 3150-2550$ (bs, $\left.\mathrm{OH}\right), 1685$ $(\mathrm{C}=\mathrm{O}) ; \delta_{\mathrm{H}}\left(400 \mathrm{MHz}, \mathrm{CDCl}_{3}\right) 3.51(2 \mathrm{H}, \mathrm{s}), 5.28(1 \mathrm{H}, \mathrm{s}), 5.57(1 \mathrm{H}, \mathrm{s}), 7.17\left(2 \mathrm{H}, \mathrm{d},{ }^{3} J=6.4 \mathrm{~Hz}\right), 7.65\left(2 \mathrm{H}, \mathrm{d},{ }^{3} J=\right.$ $6.4 \mathrm{~Hz}) ; \delta_{\mathrm{C}}\left(100.5 \mathrm{MHz}, \mathrm{CDCl}_{3}\right) 40.4\left(\mathrm{CH}_{2}\right), 117.5\left(\mathrm{CH}_{2}\right), 127.6(2 \mathrm{C}, \mathrm{CH}), 133.1\left(\mathrm{C}_{\text {quat }}\right), 137.6(2 \mathrm{C}, \mathrm{CH}), 138.9$ $\left(\mathrm{C}_{\text {quat }}\right), 139.4\left(\mathrm{C}_{\text {quat }}\right), 174.9\left(\mathrm{C}_{\text {quat }}, \mathrm{CO}\right)$.

$(\boldsymbol{E})$-3-Phenylpent-2-enoic acid $(\boldsymbol{E}-8 \mathrm{f})$ (Procedure A). Colorless solid, mp. $93^{\circ} \mathrm{C}$ [Lit. $\left.95^{\circ} \mathrm{C}[54]\right] ; v$ $\left(\mathrm{KBr} / \mathrm{cm}^{-1}\right) 3150-2550(\mathrm{bs}, \mathrm{OH}), 1698(\mathrm{C}=\mathrm{O}) ; \delta_{\mathrm{H}}\left(400 \mathrm{MHz}, \mathrm{CDCl}_{3}\right) 1.09\left(3 \mathrm{H}, \mathrm{t},{ }^{3} J=7.6 \mathrm{~Hz}, \mathrm{CH}_{3}\right), 3.13(2 \mathrm{H}$, q, $\left.{ }^{3} J=7.6 \mathrm{~Hz}\right), 6.06(1 \mathrm{H}, \mathrm{s}), 7.38-7.40(3 \mathrm{H}, \mathrm{m}), 7.46-7.47(2 \mathrm{H}, \mathrm{m}), 11.7(1 \mathrm{H}, \mathrm{s}, \mathrm{OH}) ; \delta_{\mathrm{C}}\left(100.5 \mathrm{MHz}, \mathrm{CDCl}_{3}\right)$ 13.6 $\left(\mathrm{CH}_{3}\right), 24.5\left(\mathrm{CH}_{2}\right), 115.8(\mathrm{CH}), 126.7(\mathrm{CH}, 2 \mathrm{C}), 128.6(\mathrm{CH}, 2 \mathrm{C}), 129.2(\mathrm{CH}), 140.9\left(\mathrm{C}_{\text {quat }}\right), 165.0\left(\mathrm{C}_{\text {quat }}\right)$, $171.2\left(\mathrm{C}_{\text {quat }}\right)$; and $(\boldsymbol{Z})$-3-phenylpent-2-enoic acid $(\boldsymbol{Z}-8 \mathrm{f})$ as a colorless solid, mp. $74{ }^{\circ} \mathrm{C} ; v \square\left(\mathrm{KBr} / \mathrm{cm}^{-1}\right) 3180$ 2550 (bs, OH), $1687(\mathrm{C}=\mathrm{O}) ; \delta_{\mathrm{H}}\left(400 \mathrm{MHz}, \mathrm{CDCl}_{3}\right) 1.04\left(3 \mathrm{H}, \mathrm{t},{ }^{3} J=7.2 \mathrm{~Hz}, \mathrm{CH}_{3}\right), 2.44\left(2 \mathrm{H}, \mathrm{q},{ }^{3} J=7.2 \mathrm{~Hz}\right)$, $5.86(1 \mathrm{H}, \mathrm{s}), 7.13-7.15(2 \mathrm{H}, \mathrm{m}), 7.30-7.35(3 \mathrm{H}, \mathrm{m}) ; \delta_{\mathrm{C}}\left(100.5 \mathrm{MHz}, \mathrm{CDCl}_{3}\right) 12.0\left(\mathrm{CH}_{3}\right), 33.8\left(\mathrm{CH}_{2}\right), 115.3$ $(\mathrm{CH}), 127.0(\mathrm{CH}, 2 \mathrm{C}), 127.8(\mathrm{CH}), 128.0(\mathrm{CH}, 2 \mathrm{C}), 139.8\left(\mathrm{C}_{\text {quat }}\right), 163.8\left(\mathrm{C}_{\text {quat }}\right), 170.0\left(\mathrm{C}_{\text {quat }}\right)$.

(E)-4-Phenylbut-2-enoic acid $(\mathbf{8 g})$ (procedure A). Beige solid, mp. $96^{\circ} \mathrm{C}$ [Lit. $\left.97^{\circ} \mathrm{C}[55]\right] ; v_{\max }\left(\mathrm{KBr} / \mathrm{cm}^{-1}\right)$ 3054, $1702(\mathrm{C}=\mathrm{O}), 1409,1218(\mathrm{C}-\mathrm{O}), 977,914,745,692 ; \delta_{\mathrm{H}}\left(400 \mathrm{MHz}, \mathrm{DMSO}-\mathrm{d}^{6}\right) 3.23\left(2 \mathrm{H}, \mathrm{d},{ }^{3} J=7.2 \mathrm{~Hz}\right)$, $6.28\left(1 \mathrm{H}, \mathrm{dd},{ }^{3} \mathrm{~J}=16.4 \mathrm{~Hz},{ }^{3} \mathrm{~J}=7.2 \mathrm{~Hz}\right), 6.46\left(1 \mathrm{H}, \mathrm{d},{ }^{3} J=16.4 \mathrm{~Hz}\right), 7.20(1 \mathrm{H}, \mathrm{m}), 7.28\left(2 \mathrm{H}, \mathrm{dd},{ }^{3} J=7.2 \mathrm{~Hz},{ }^{3} \mathrm{~J}=\right.$ $7.2 \mathrm{~Hz}), 7.38\left(2 \mathrm{H}, \mathrm{d},{ }^{3} J=7.2 \mathrm{~Hz}\right) ; \delta_{\mathrm{H}}\left(400 \mathrm{MHz}, \mathrm{CDCl}_{3}\right) 3.30\left(2 \mathrm{H}, \mathrm{d},{ }^{3} J=8.4 \mathrm{~Hz}\right), 6.29\left(1 \mathrm{H}, \mathrm{dd},{ }^{3} J=15.6 \mathrm{~Hz},{ }^{3} J\right.$ $=8.4 \mathrm{~Hz}), 6.52\left(1 \mathrm{H}, \mathrm{d},{ }^{3} J=15.6 \mathrm{~Hz}\right), 7.25(1 \mathrm{H}, \mathrm{m}), 7.32(2 \mathrm{H}, \mathrm{m}), 7.39(2 \mathrm{H}, \mathrm{m}) ; \delta_{\mathrm{C}}\left(100.5 \mathrm{MHz}, \mathrm{DMSO}-\mathrm{d}^{6}\right) 38.3$ $\left(\mathrm{CH}_{2}\right), 123.6(\mathrm{CH}), 126.4(2 \mathrm{C}, \mathrm{CH}), 127.9(\mathrm{CH}), 129.1(2 \mathrm{C}, \mathrm{CH}), 132.7(\mathrm{CH}), 137.1\left(\mathrm{C}_{\text {quat }}\right), 173.2(\mathrm{CO}) ; \delta_{\mathrm{C}}$ $\left.\left(100.5 \mathrm{MHz}, \mathrm{CDCl}_{3}\right) 38.1\left(\mathrm{CH}_{2}\right), 120.9(\mathrm{CH}), 126.3(2 \mathrm{C}, \mathrm{CH}), 127.7 \mathrm{CH}\right), 128.6(2 \mathrm{C}, \mathrm{CH}), 134.0(\mathrm{CH}), 136.6$ $\left(\mathrm{C}_{\text {quat }}\right), 177.8\left(\mathrm{C}_{\text {quat }}, \mathrm{CO}\right)$.

Ethyl (E)-3-anthran-1-ylbut-2-enoate (10b). A mixture of 1-acetylanthracene (300 mg, $1.36 \mathrm{mmol})$, phosphorane $2 \mathrm{a}(760 \mathrm{mg}, 2.18 \mathrm{mmol})$, and benzoic acid $(30 \mathrm{mg}, 0.25 \mathrm{mmol})$ in benzene $(8 \mathrm{~mL})$ was heated at $80^{\circ} \mathrm{C}$ for $12 \mathrm{~h}$. After $3 \mathrm{~h}$, additional $2 \mathrm{a}(300 \mathrm{mg}, 0.87 \mathrm{mmol})$ was added. The cooled reaction mixture was concentrated in vacuo and subjected to column chromatography on silica gel (ether/ $\mathrm{CHCl}_{3} /$ hexane 1:3:3) to give $\mathbf{1 0 b}$ $(25 \mathrm{mg}, 6 \%)$ as a colorless oil. $v\left(\right.$ neat $\left./ \mathrm{cm}^{-1}\right) 1720(\mathrm{C}=\mathrm{O}), 1634,1170 ; \delta_{\mathrm{H}}\left(400 \mathrm{MHz}, \mathrm{CDCl}_{3}\right) 1.35\left(3 \mathrm{H}, \mathrm{t},{ }^{3} \mathrm{~J}=\right.$ $7.2 \mathrm{~Hz}), 2.70\left(3 \mathrm{H}, \mathrm{d},{ }^{4} J=1.2 \mathrm{~Hz}\right), 4.28\left(2 \mathrm{H}, \mathrm{q},{ }^{3} J=7.2 \mathrm{~Hz}\right), 6.07\left(1 \mathrm{H}, \mathrm{q},{ }^{4} J=1.2 \mathrm{~Hz}\right), 7.25\left(1 \mathrm{H}, \mathrm{d},{ }^{3} J=6.8 \mathrm{~Hz}\right)$, $7.42\left(1 \mathrm{H}, \mathrm{dd},{ }^{3} \mathrm{~J}=8.4 \mathrm{~Hz},{ }^{3} \mathrm{~J}=6.8 \mathrm{~Hz}\right), 7.47-7.48(1 \mathrm{H}, \mathrm{m}), 7.95-8.00(3 \mathrm{H}, \mathrm{m}), 8.41(1 \mathrm{H}, \mathrm{s}), 8.44(1 \mathrm{H}, \mathrm{s}) ; \delta_{\mathrm{C}}$ $\left(100.5 \mathrm{MHz}, \mathrm{CDCl}_{3}\right) 14.3\left(\mathrm{CH}_{3}\right), 21.6\left(\mathrm{CH}_{3}\right), 60.0\left(\mathrm{OCH}_{2}\right), 120.6(\mathrm{CH}), 123.5(\mathrm{CH}), 124.2(\mathrm{CH}), 124.6(\mathrm{CH})$, 125.6 (CH), $125.8(\mathrm{CH}), 126.8(\mathrm{CH}), 127.3\left(\mathrm{C}_{\text {quat }}\right), 127.6\left(\mathrm{C}_{\text {quat }}\right), 127.9(\mathrm{CH}), 128.5(2 \mathrm{C}, \mathrm{CH}), 128.7\left(\mathrm{C}_{\text {quat }}\right)$, $131.5\left(\mathrm{C}_{\text {quat }}\right), 131.8\left(\mathrm{C}_{\text {quat }}\right), 142.1\left(\mathrm{C}_{\text {quat }}\right), 166.8\left(\mathrm{C}_{\text {quat }}, \mathrm{CO}\right)$.

For the preparation of 12a-12c, general procedure A was used. After acidification of the reaction medium after completion of the reaction, the aqueous phase was extracted with $\mathrm{CHCl}_{3}$. The organic phase was dried over $\mathrm{MgSO}_{4}$, concentrated in vacuo and the residue was filtered over a small layer of silica gel (ether $/ \mathrm{CHCl}_{3} 1: 1$ ).

5,9-Dimethyldeca-2,8-dienoic acid (12a) [56] as a colorless oil; $v_{\max }$ (neat $\left./ \mathrm{cm}^{-1}\right) 3100-2550$ (bs, OH), 2917, $1695(\mathrm{C}=\mathrm{O}), 1648,1428,1282,983,938,686 ; \delta_{\mathrm{H}}\left(3 \mathrm{H}, \mathrm{d},{ }^{3} J=6.8 \mathrm{~Hz}\right), 0.95(1 \mathrm{H}, \mathrm{m}), 1.10(1 \mathrm{H}, \mathrm{m}), 1.35(1 \mathrm{H}$, $\mathrm{m}), 1.59\left(3 \mathrm{H}, \mathrm{d},{ }^{4} J=0.8 \mathrm{~Hz}\right), 1.68\left(3 \mathrm{H}, \mathrm{d},{ }^{4} J=0.8 \mathrm{~Hz}\right), 1.90-2.07(2 \mathrm{H}, \mathrm{m}), 2.22-2.24(2 \mathrm{H}, \mathrm{m}), 5.04(1 \mathrm{H}, \mathrm{m})$, $5.82\left(1 \mathrm{H}, \mathrm{d},{ }^{3} \mathrm{~J}=15.6 \mathrm{~Hz}\right), 7.07(1 \mathrm{H}, \mathrm{m}) ; \delta_{\mathrm{C}}\left(100.5 \mathrm{MHz}, \mathrm{CDCl}_{3}\right) 17.7,19.5,32.0,25.5,25.9,36.7,39.7,121.8$, $124.3,131.6,151.3,171.9(\mathrm{CO})$.

(E)-Dec-2-enoic acid (12b) [57]. Colorless oil; $v_{\max }\left(\right.$ neat $\left./ \mathrm{cm}^{-1}\right) 1698(\mathrm{C}=\mathrm{O}), 1660,1422,1310,1300,980$, 
940; $\delta_{\mathrm{H}}\left(400 \mathrm{MHz}, \mathrm{CDCl}_{3}\right) 0.83\left(3 \mathrm{H}, \mathrm{t},{ }^{3} J=5.6 \mathrm{~Hz}, \mathrm{CH}_{3}\right), 1.22(\mathrm{~m}), 1.41(2 \mathrm{H}, \mathrm{m}), 2.17(2 \mathrm{H}, \mathrm{m}), 5.76\left(1 \mathrm{H}, \mathrm{d},{ }^{3} J\right.$ $=15.2 \mathrm{~Hz}), 7.03\left(1 \mathrm{H}, \mathrm{dt},{ }^{3} J=15.2 \mathrm{~Hz},{ }^{3} J=5.6 \mathrm{~Hz}\right) ; \delta_{\mathrm{C}}\left(100.5 \mathrm{MHz}, \mathrm{CDCl}_{3}\right) 14.0\left(\mathrm{CH}_{3}\right), 22.6\left(\mathrm{CH}_{2}\right), 27.8\left(\mathrm{CH}_{2}\right)$, $29.0\left(\mathrm{CH}_{2}\right), 29.1\left(\mathrm{CH}_{2}\right), 31.7\left(\mathrm{CH}_{2}\right), 32.3\left(\mathrm{CH}_{2}\right), 120.6(\mathrm{CH}), 152.4(\mathrm{CH}), 172.1\left(\mathrm{C}_{\text {quat, }}, \mathrm{CO}\right)$; MS (EI, $\left.70 \mathrm{eV}\right) \mathrm{m} / \mathrm{z}$ (\%) $170\left(\mathrm{M}^{+}, 13\right), 73(65)$.

3-Cyclohexylpropenoic acid (12c). Colorless solid, mp. $53^{\circ} \mathrm{C}\left[\right.$ Lit. $\left.57^{\circ} \mathrm{C}-58^{\circ} \mathrm{C}[58]\right], v_{\max }$ (neat) $3400-2550$ (bs, OH), 2931, 2856, $1699(\mathrm{C}=\mathrm{O}) ; \delta_{\mathrm{H}}\left(400 \mathrm{MHz}, \mathrm{CDCl}_{3}\right) 1.00-2.05(10 \mathrm{H}, \mathrm{m}), 2.20(1 \mathrm{H}, \mathrm{m}), 5.69\left(1 \mathrm{H}, \mathrm{dd},{ }^{3} J=\right.$ $\left.16.0 \mathrm{~Hz},{ }^{4} J=2.0 \mathrm{~Hz}\right), 6.95\left(1 \mathrm{H}, \mathrm{dd},{ }^{3} J=16.0 \mathrm{~Hz},{ }^{3} J=6.8 \mathrm{~Hz}\right), 9.40-10.00(1 \mathrm{H}, \mathrm{bs}, \mathrm{OH}) ; \delta_{\mathrm{C}}(100.5 \mathrm{MHz}$, $\left.\mathrm{CDCl}_{3}\right)$ 25.6, 25.9, 31.6, 40.5, 118.5, 158.2, 172.9.

\subsection{General Procedure B}

Phenylpropiolic acid (13b). To a mixture of benzaldehyde (1f, $763 \mathrm{mg}, 7.2 \mathrm{mmol}$ ) and ethoxycarbonylbromomethylidenetriphenylphosphorane (2b, $4.0 \mathrm{~g}, 9.4 \mathrm{mmol})$ was given aq. $\mathrm{NaOH}$ solution $(10 \mathrm{w} \%, 1.8 \mathrm{~g} \mathrm{NaOH}$ in $18 \mathrm{~mL} \mathrm{H}_{2} \mathrm{O}$ ) and the resulting suspension was stirred at $85^{\circ} \mathrm{C}$ for $16 \mathrm{~h}$. Triphenylphosphine oxide was filtered off the cooled solution. Thereafter, the filtrate was acidified carefully with $15 \mathrm{w} \%$ aq. $\mathrm{HCl}$. The resulting suspension was cooled and filtered. The solid obtained was dried in air to give phenylpropiolic acid $(\mathbf{1 3 b}, 589 \mathrm{mg}, 56 \%)$ as a colorless solid. An analytical sample gave mp. $133^{\circ} \mathrm{C}\left[\right.$ Lit. $\left.135^{\circ} \mathrm{C}-137^{\circ} \mathrm{C}[41]\right]-v_{\max }\left(\mathrm{KBr} / \mathrm{cm}^{-1}\right) 3200-2500$ (bs, OH), 2238, $2201(\mathrm{C} \equiv \mathrm{C}), 1670(\mathrm{C}=\mathrm{O}), 1489,1417,1305,1208(\mathrm{C}-\mathrm{O}), 919,753,683,610,533,509 ; \delta_{\mathrm{H}}(400$ $\left.\mathrm{MHz}, \mathrm{CDCl}_{3}\right) 7.39-7.42(2 \mathrm{H}, \mathrm{m}), 7.46-7.48(1 \mathrm{H}, \mathrm{m}), 7.60-7.62(2 \mathrm{H}, \mathrm{m}), 10.1(\mathrm{bs}, \mathrm{OH}) ; \delta_{\mathrm{C}}(100.5 \mathrm{MHz}$, $\left.\mathrm{CDCl}_{3}\right) 80.2\left(\mathrm{C}_{\text {quat }}\right), 88.8\left(\mathrm{C}_{\text {quat }}\right), 119.1\left(\mathrm{C}_{\text {quat }}\right), 128.8(2 \mathrm{C}, \mathrm{CH}), 131.1(\mathrm{CH}), 133.3(2 \mathrm{C}, \mathrm{CH}), 158.4\left(\mathrm{C}_{\text {quat }}, \mathrm{CO}\right)$; MS (EI, $70 \mathrm{eV}) \mathrm{m} / z(\%) 146\left(\mathrm{M}^{+}\right)(100), 76(30)$.

4-Methoxyphenylpropiolic acid (13a) (Procedure B). Pale yellow solid, mp. $143^{\circ} \mathrm{C}$ [Lit. 144 $\left.{ }^{\circ} \mathrm{C}[59]\right]$; $v_{\max }$ $\left(\mathrm{KBr} / \mathrm{cm}^{-1}\right) 2197(\mathrm{C} \equiv \mathrm{C}), 1671(\mathrm{C}=\mathrm{O}), 1602(\mathrm{C}=\mathrm{C}), 1511,1417,1322,1297,1255,1213,1168,1028,923,832$, $799,609,565,539 ; \delta_{\mathrm{H}}\left(400 \mathrm{MHz}, \mathrm{CDCl}_{3}\right) 3.84\left(3 \mathrm{H}, \mathrm{s}, \mathrm{OCH}_{3}\right), 6.89\left(2 \mathrm{H}, \mathrm{d},{ }^{3} J=8.8 \mathrm{~Hz}\right), 7.56\left(2 \mathrm{H}, \mathrm{d},{ }^{3} J=8.8\right.$ $\mathrm{Hz}) ; \delta_{\mathrm{C}}\left(100.5 \mathrm{MHz}, \mathrm{CDCl}_{3}\right) 55.5\left(\mathrm{OCH}_{3}\right), 79.6\left(\mathrm{C}_{\text {quat }}\right), 90.0\left(\mathrm{C}_{\text {quat }}\right), 110.8\left(\mathrm{C}_{\text {quat }}\right), 114.4(2 \mathrm{C}, \mathrm{CH}), 135.3(2 \mathrm{C}$, $\mathrm{CH}), 158.4\left(\mathrm{C}_{\text {quat }}\right), 161.9\left(\mathrm{C}_{\text {quat }}, \mathrm{CO}\right) ; \delta_{\mathrm{C}}\left(100.5 \mathrm{MHz}, \mathrm{DMSO}-\mathrm{d}^{6}\right) 55.8\left(\mathrm{OCH}_{3}\right), 81.5\left(\mathrm{C}_{\text {quat }}\right), 85.9\left(\mathrm{C}_{\text {quat }}\right), 110.9$ $\left(\mathrm{C}_{\text {quat }}\right), 115.1(2 \mathrm{C}, \mathrm{CH}), 135.1(2 \mathrm{C}, \mathrm{CH}), 155.1\left(\mathrm{C}_{\text {quat }}\right), 161.6\left(\mathrm{C}_{\text {quat }}, \mathrm{CO}\right)$.

3-Chlorophenylpropiolic acid (13c) (Procedure B). Colorless solid, mp. $145^{\circ} \mathrm{C}$ [Lit. $144^{\circ} \mathrm{C}$ [60]]; $v_{\max }$ $\left(\mathrm{KBr} / \mathrm{cm}^{-1}\right) 3200-2500(\mathrm{bs}, \mathrm{OH}), 2960,2586,2217(\mathrm{C} \equiv \mathrm{C}), 1683(\mathrm{C}=\mathrm{O}), 1475,1420,1310,1208,877,784$, 674, 611, 519; $\delta_{\mathrm{H}}\left(400 \mathrm{MHz}, \mathrm{CDCl}_{3}\right) ; 9.0(1 \mathrm{H}, \mathrm{bs}, \mathrm{OH}), 7.31-7.34(1 \mathrm{H}, \mathrm{m}), 7.44-7.49(2 \mathrm{H}, \mathrm{m}), 7.58(1 \mathrm{H}, \mathrm{s})$; $\delta_{\mathrm{C}}\left(100.5 \mathrm{MHz}, \mathrm{DMSO}-\mathrm{d}^{6}\right) 81.0\left(\mathrm{C}_{\text {quat }}\right), 86.3\left(\mathrm{C}_{\text {quat }}\right), 121.0\left(\mathrm{C}_{\text {quat }}\right), 129.9(\mathrm{CH}), 131.3(2 \mathrm{C}, \mathrm{CH}), 132.8(\mathrm{CH})$, $134.5\left(\mathrm{C}_{\text {quat }}\right), 157.4\left(\mathrm{C}_{\text {quat }}\right)$.

3,4-Dimethoxyphenylpropiolic acid (13d) (Procedure B). Colorless solid, mp. $154^{\circ} \mathrm{C}\left[\right.$ Lit. $152^{\circ} \mathrm{C}-153^{\circ} \mathrm{C}$ [61]]; $v_{\max }\left(\mathrm{KBr} / \mathrm{cm}^{-1}\right) 3200-2500(\mathrm{bs}, \mathrm{OH}), 2937,2837,2595,2202(\mathrm{C} \equiv \mathrm{C}), 1684(\mathrm{C}=\mathrm{O}), 1625,1596,1517$, $1312,1252,1165,1140,1022,975,839,810,723,579,540 . \delta_{\mathrm{H}}\left(400 \mathrm{MHz}, \mathrm{CDCl}_{3}\right) 3.89\left(3 \mathrm{H}, \mathrm{s}, \mathrm{OCH}_{3}\right), 3.92$ $\left(3 \mathrm{H}, \mathrm{s}, \mathrm{OCH}_{3}\right), \delta_{\mathrm{C}}\left(100.5 \mathrm{MHz}, \mathrm{CDCl}_{3}\right) 79.4\left(\mathrm{C}_{\text {quat }}\right), 89.8\left(\mathrm{C}_{\text {quat }}\right), 110.9\left(\mathrm{C}_{\text {quat }}\right), 111.0(\mathrm{CH}), 115.4(\mathrm{CH}), 127.7$ $(\mathrm{CH}), 148.8\left(\mathrm{C}_{\text {quat }}\right), 151.9\left(\mathrm{C}_{\text {quat }}\right), 157.9\left(\mathrm{C}_{\text {quat }}\right)$.

\section{Results and Discussion}

When a mixture of benzaldehyde $\mathbf{1}$ and ethoxymethylidenetriphenylphosphorane (2a, 1.3 eq.) is heated in a 10 $\mathrm{w} \%$ aq. $\mathrm{NaOH}$ solution, sodium cinnamates are formed by Wittig olefination and subsequent ester hydrolysis. Upon neutralization of the aqueous solutions with $10 \mathrm{w} \%-15 \mathrm{w} \%$ aq. $\mathrm{HCl}$, cinnamic acids 3 are obtained by easy filtration and drying of the solids in air. With this procedure, cinnamic acids $\mathbf{3}$ could be synthesized (Table 1).

Even solid 3e can be filtered off from the neutralized solution easily, as the anilinic nitrogen in $\mathbf{3 e}$ is not basic enough to be protonated upon careful neutralization of the reaction mixture. Most of the cinnamic acids are produced in good $E$-selectivity. However, for phenylpenta-2,5-dienoic acid (3p), 2,4-dimethoxycinnamic acid (3g), 2,5-dimethoxycinnamic acid (3h) and especially 3-bromo-2,5-dimethoxycinnamic acid (3i) anoticable quantity of $Z$-isomer is formed.

4-Nitrobenzaldehyde (1w) is not an adequate substrate for this one-pot procedure, even though 4-nitrobenzaldehyde (1w) and ethoxymethylidenetriphenylphosphorane (2a) give the Wittig product3wfacilely, when the reaction is performed in chloroform (Scheme 1). Also, the ester hydrolysis of $\mathbf{3 w}$ in $10 \mathrm{w} \%$ aq. NaOH proceeds 
Table 1. Substituted cinnamic acids via one pot Wittig olefination-hydrolysis reaction.

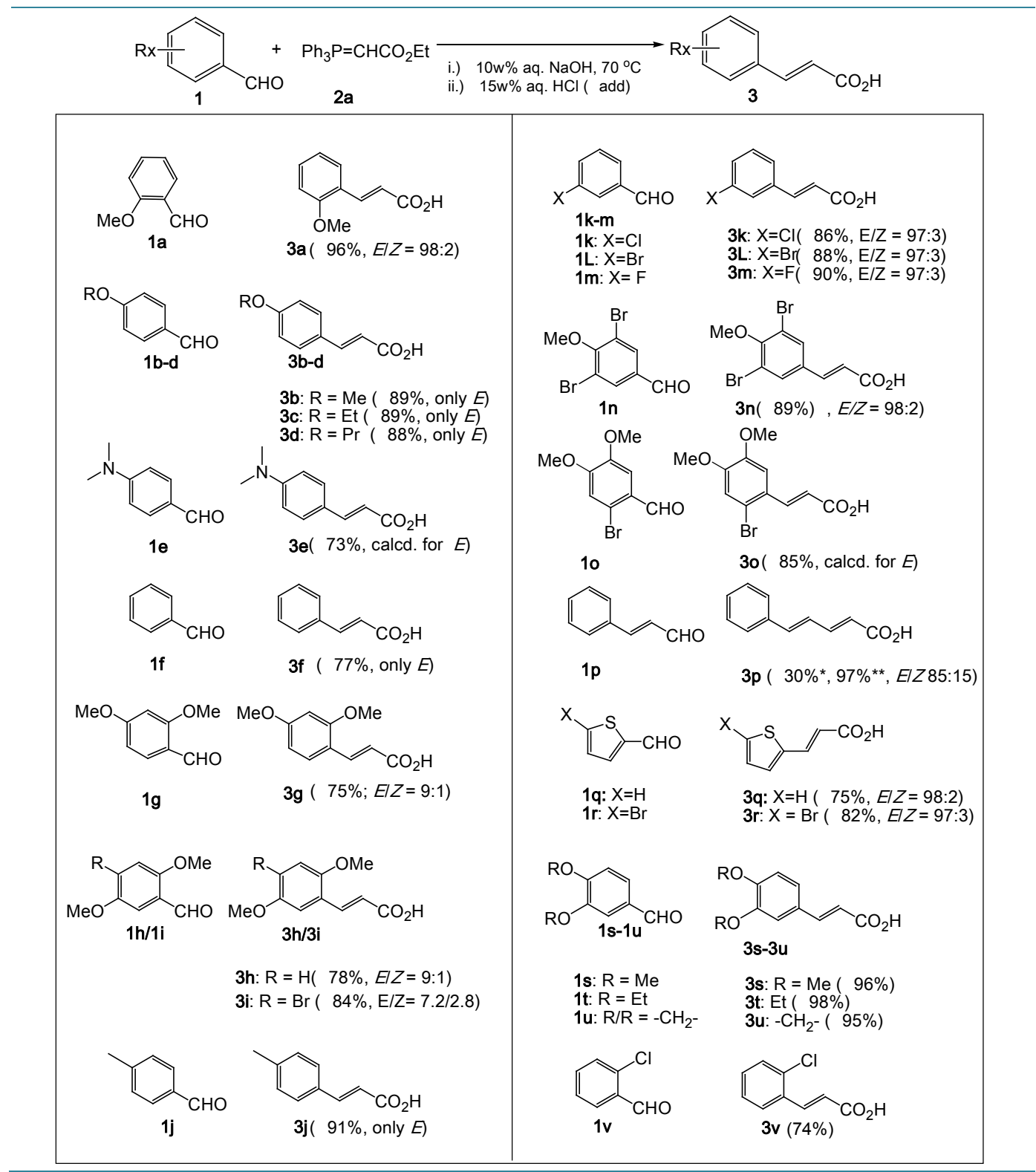

*no additive; **tetramethylammonium bromide $\left(0.15\right.$ eq. $\left.\mathrm{Bu}_{4} \mathrm{NBr}\right)$ addad.

readily, and 3x is obtained, which can be filtered off and dried after acidification of the reaction solution. Yet, in $10 \mathrm{w} \%$ aq. $\mathrm{NaOH}, 4$-nitrobenzaldehyde (1w) undergoes a rapid Cannizzaro reaction, which would be expected to be the general competing pathway for benzaldehydes in aqueous basic media. However, with the exception of the nitro-substituted benzaldehydes, Cannizzaro is not noted to be a main side reaction for the benzaldehydes used. The reason for this may be that the Wittig olefination ensues rapidly in the lipotropic droplets formed by themixture of benzaldehyde and phosphorane, initially suspended in the aqueous solution. In the case of 4-nitrobenzaldehyde (1r) in $10 \mathrm{w} \%$ aq. $\mathrm{NaOH}$, a deep red homogeneous solution is produced immediately, from which one of the Cannizzaro products, 4-nitrobenzoic acid (4), can be obtained facilely by extraction of the side product with $\mathrm{CH}_{2} \mathrm{Cl}_{2}$ and acidification of the aqueous phase with subsequent extraction of the phase and crystallization of the obtained product [62] [63]. Cannizaro reaction can also be noted for 2-nitrobenzaldehyde under the conditions, and the 2-nitrobenzoic acid can be obtained easily.

In the case of 1x (Figure 1), only (Z)-p-benzyloxycinnamic acid (3y) was obtained as the cinnamic acid 

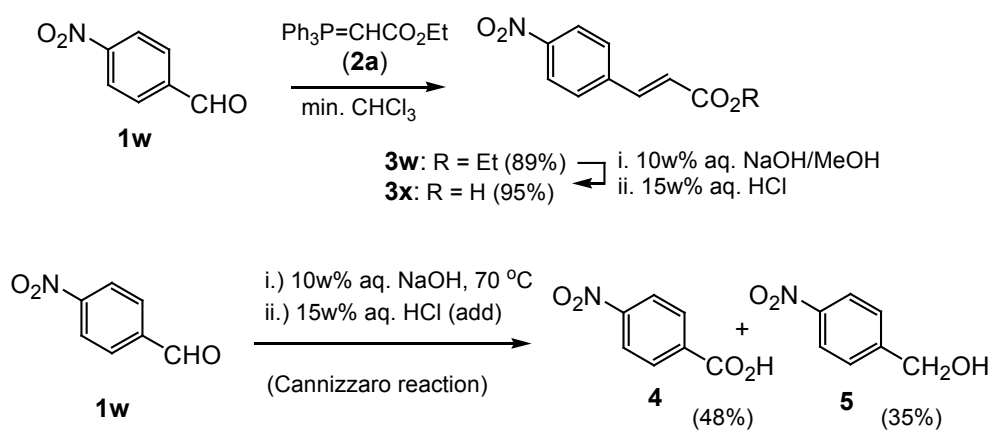

Scheme 1. Comparison of the sequential Wittig olefination-hydrolysis reaction of 4-nitrobenzaldehyde ( $1 \mathrm{w}$ ) leading to cinnamic acid $3 \mathrm{x}$ and the reaction under one pot conditions leading to Cannizzaro products 4 and 5 .

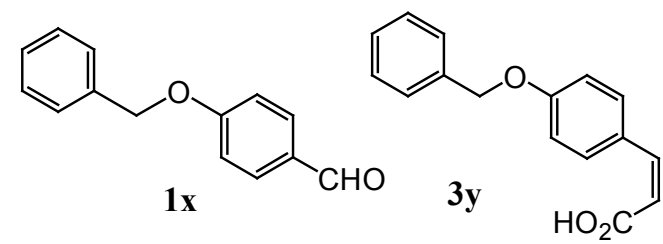

Figure 1. Reduction of alkenes carrying $O$-benzyl esterand/or $O$-benzyl ether functions.

fraction. The $E$-isomer could not be detected. At $70^{\circ} \mathrm{C}$, triphenylphosphine oxide and the Wittig product develop liquid hydrophobic micelles. Ester hydrolysis takes place at the micelle boundary, and it may be that the ethyl $(E)$ - $p$-benzyloxycinnamate, more easily forming intermolecular layers within the micelle, is not as readily accessible as the $Z$-isomer. It must also be noticed that under the strongly basic conditions, the benzyloxy function is not sufficiently stable and is severed to provide the hydroxy-substituted compound. That micelles play a role in the ester hydrolysis can be seenin alkyl cinnamates with larger residues, such as in ethyl phenylpenta-2,4-dienoate, where the addition of tetramethylammonium bromide $\left[\left(\mathrm{CH}_{3}\right)_{4} \mathrm{NBr}\right]$ leads to more yield in phenylpenta-2,4-dienoic acid (3p).

In cases, where the arylcarbaldehydes undergo Wittig reaction with alkoxycarbonylmethylidene phosphoranes slowly and only at higher temperatures at which the phosphoranes are already hydrolysed, a variant of the reaction protocol can be employed. First, the aldehyde is reacted with the phosphorane under solventless conditions at higher temperatures. Thereafter, $10 \mathrm{w} \%$ aq. $\mathrm{NaOH}$ is added to the reaction mixture, and the reaction is completed. 9-Anthranylcarbaldehyde (1t) represents such a case, where the aldehyde undergoes Wittig olefination only sluggishly due to the steric congestion around the carbaldehyde function. 1t can be reacted with $\mathbf{2 a}$ under solventless conditions at $130^{\circ} \mathrm{C}$ [64]. Subsequent addition of aq. $\mathrm{NaOH}$ to the reaction and heating of the mixture gives the 3-(anthran-9-yl)propenoic acid (3z) in 85\% yield (Scheme 2). It must be noted that upon cooling of the reaction, sodium 3-(anthran-9-yl)propenoate (3z-Na) crystallizes in the aqueous solution, and the resulting solid, which is comprised of the sodium salt of the acid as well as triphenylphosphine oxide, must be washed diligently with hot water upon filtration of the triphenylphosphine oxide. The precipitation was also noted in the hydrolysis of isolated ethyl anthranylpropenoate (3z-Et, $\mathrm{R}=\mathrm{Et})$ in $10 \mathrm{w} \%$ aq. $\mathrm{NaOH}$. In that case, the precipitate can be treated directly with aq. $\mathrm{HCl}$ to yield anthranylpropenoic acid (3z) as a filterable precipitate.

The same solventless procedure was followed, when reacting 4-hydroxybenzaldehyde (1z) with phosphorane 2a at $140^{\circ} \mathrm{C}$ for $90 \mathrm{~min}$. Thereafter, the mixture was taken up in $10 \mathrm{w} \%$ aq. $\mathrm{NaOH}$ and stirred at $\mathrm{rt}$ for $30 \mathrm{~min}$., after which the triphenylphosphine oxide was filtered off, and the filtrate acidified with half-conc. aq. $\mathrm{HCl}$ to give 4-hydroxycinnamic acid (6, $p$-coumaric acid) (Scheme 3). Previously, 4-hydroxycinnamic acid had been prepared by Horner Emmons olefination [65] with a subsequent, second hydrolysis step and via Wittig reaction with a polymer-bound Wittig reagent and a subsequent hydrolysis step [66].

Acetophenones 7 have been subjected successfully to the one-pot Wittig olefination-hydrolysis, too (Table 2). In these cases, a mixture of phosphorane 2a and acetophenone 7 was heated, and thereafter an aq. NaOH solution was added. A workup identical to that described for the benzaldehydes provided the products as mixtures of 


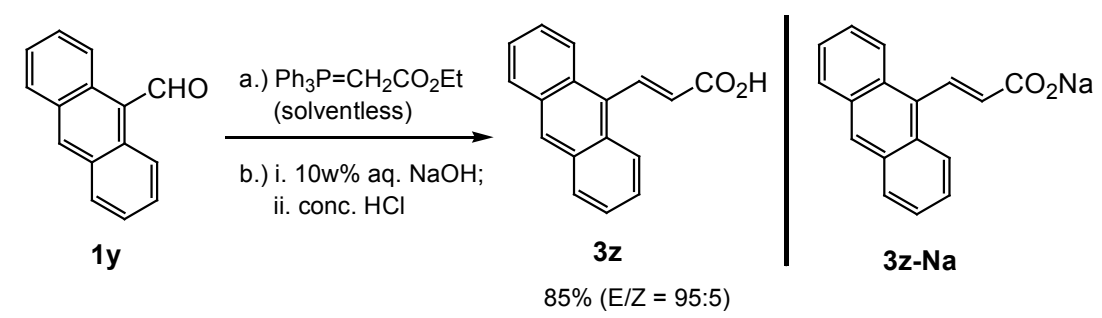

Scheme 2. Solventless Wittig reactionand subsequent hydrolysis without work-up of $3 \mathrm{z}$.

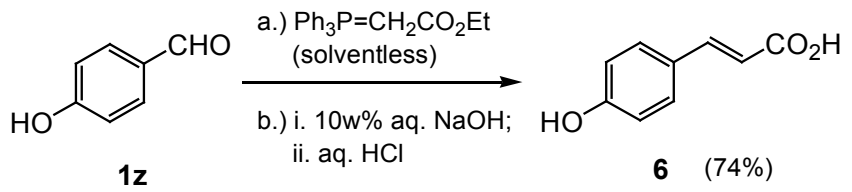

Scheme 3. Preparation of $p$-coumaric acid (6) by solventless Wittig-olefination with basic extractive work-up.

Table 2. One pot Wittig reaction and hydrolysis with acetophenone as starting material.
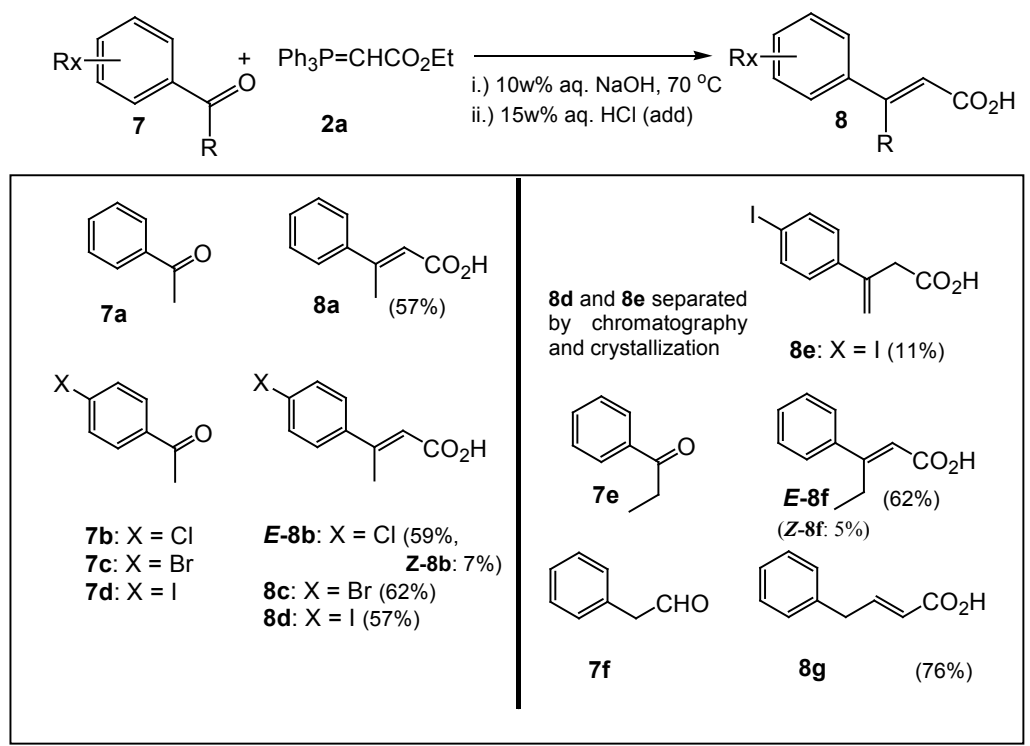

$E / Z$-isomers. The $E$-isomer could be obtained in pure form by recrystallization from $1: 1 \mathrm{v} / \mathrm{v} \mathrm{CH}_{2} \mathrm{Cl}_{2} / \mathrm{hexane}$ In order to obtain both isomers in pure form, the mixture was subjected to chromatography on silica gel (hexane/EtOAc 2:1). The reaction is dependent on the steric bulkiness of the ketone, where bulkiness in the ketone leads to a sterically congested transition state in the Wittig olefination. Thus, 1-acetylanthracene (9) does not react under the conditions to the respective acid at all. 9 reacts with phosphorane $\mathbf{2 a}$ sluggishly, and produces butenoate 10b after $12 \mathrm{~h}$ in refluxing chloroform in the presence of benzoic acid as acid catalyst only in trace amounts. Under the conditions of the one-pot Wittig olefination-ester hydrolysis, much of the phosphorane 2a hydrolyses before undergoing Wittig olefination with 9 (Scheme 4).

Alkanals and alicyclic carbaldehydes $\mathbf{1 1}$ have also been reacted in a one-pot Wittig olefination-hydrolysis reaction. The yields of the corresponding unsaturated acids $\mathbf{1 2}$ were slightly lower than for aromatic aldehydes. Also, the obtained carboxylic acids could not be purified simply by crystallization through addition of hydrochloric acid to the reaction mixture. Rather, they have to be extracted after the acidification of the reaction mixture and subjected to column chromatography on silica gel (Scheme 5).

When a mixture of (substituted) benzaldehyde $\mathbf{1}$ and ethoxybromomethylidenetriphenylphosphorane (2b, 1.3 


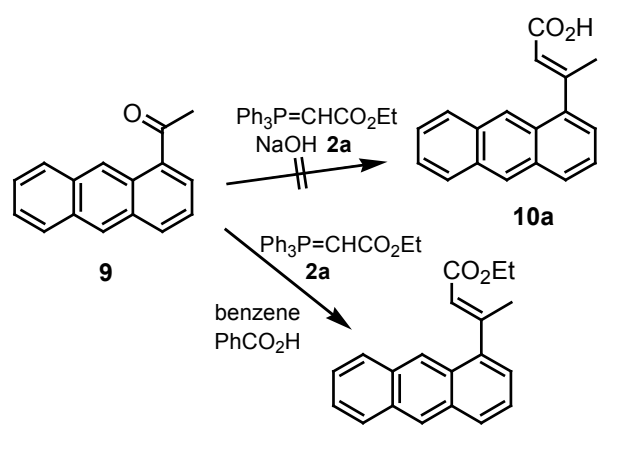

$10 \mathrm{~b}$ trace $(12 \mathrm{~h})$

Scheme 4. Wittig reaction of 1-acetylanthracene (9) with phosphorane.

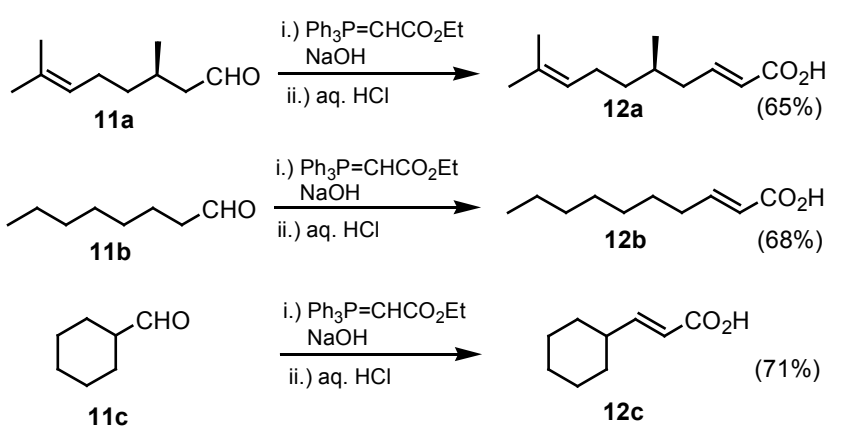

Scheme 5. One-pot transformation of alkanals and alicyclic aldehydes.

Table 3. Arylpropiolic acids from benzaldehydes in one pot synthesis in aq. solutions.

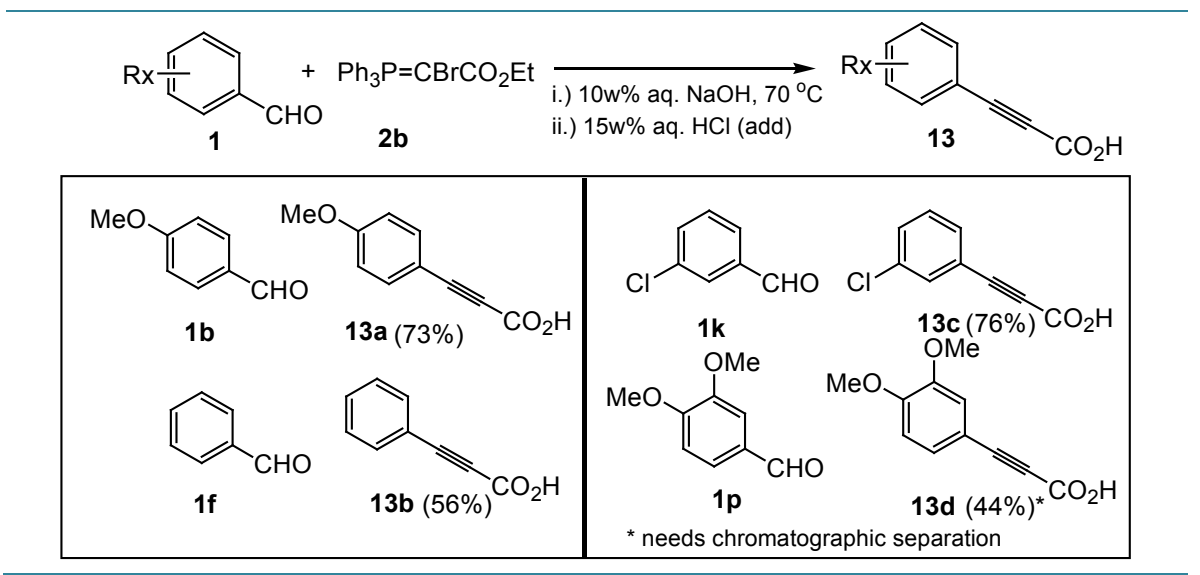

eq.) is heated in a $10 \mathrm{w} \%$ aq. $\mathbf{~ N a O H}$ solution, arylpropiolic acids $\mathbf{1 3}$ can be isolated upon careful neutralization of the aqueous solution (Table 3). In this case, the Wittig olefination is followed by ester hydrolysis and base catalysed dehydrobromination. Phenylpropiolic acids are usually prepared by bromination and dehydrobromination of cinnamic acids [60] or by Sonogashira coupling of haloarenes with propiolates [67]. In the present communication, we are showing a facile one-pot procedure to phenylpropiolic acids with a simple extractive workup. For certain derivatives, cinnamic acids have been found as side-products. Nevertheless, the procedure in its yields compares well with that of Chenault and Dupin [59], who employ ethoxyiodomethylidenetriphenylphosphorane in $\mathrm{Na}_{2} \mathrm{CO}_{3} /$ methanol, followed by subsequent acid catalysed hydrolysis of the phenylpropiolates.

\section{Conclusions}

We have developed two simple procedures from benzaldehydes to cinnamic acids and to phenylpropiolic acids, 
respectively, utilizing a modified Wittig-olefination protocol. The reactions and the work-up are performed without any organic solvents in most cases here described. Yields are reproducibly good. The one-pot procedure of olefination and ester hydrolysis can be utilized with alkanals and acetophenones, also. Here, however, chromatographic separation of the products is necessary in some cases.

In former times, the Wittig olefination reaction has not been viewed as a green reaction due to its poor atomeconomy. Recently, however, a dedicated effort has seen the development of numerous methods to recycle the toxic triphenylphosphine oxide [17] [68]-[70] that joins older methods [71], and some of them already established in industrial processes [17] [72]. The reaction procedure described above allows for an efficient separation of triphenylphosphine oxide from the mixture upon completion of the reaction by simple filtration. Then, triphenylphosphine oxide is dried and stored and can be recycled upon accumulation from a larger number of Wittig reactions.

\section{References}

[1] Korošec, B., Sova, M., Turk, S., Kraševec, N., Novak, M., Lah, L., Stojan, J., Podobnik, B., Berne, S., Zupanec, N., Bunc, M., Gobec, S. and Komel, R. (2014) Antifungal Activity of Cinnamic Acid Derivatives Involves Inhibition of Benzoate 4-Hydroxylase (CYP53). Journal of Applied Microbiology, 116, 955-966. http://doi/10.1111/jam.12417

[2] Sova, M. (2012) Antioxidant and Antimicrobial Activities of Cinnamic Acid Derivatives. Mini Reviews in Medicinal Chemistry, 12, 749-767. http://doi/10.2174/138955712801264792

[3] Breton, L., Girerd, F. and Renault, B., Societe L'Oreal (1997) FR 97 16180. Appl. December 19th, 1997.

[4] Cirigliano, M.C., Franke, W.C., Kemly, M.M., McKenna, R.T. and Rothenberg, P.J., Lipton, Div. of Conopco, Inc. (1997) US Pat. 6036986. Appl. October 28th, 1997.

[5] Shahidi, F. and Zhong, Y. (2010) Novel Antioxidants in Food Quality Preservation and Health Promotion. European Journal of Lipid Science and Technology, 112, 930-940. http://dx.doi.org/10.1002/ejlt.201000044

[6] Szmant, H.H. (1989) Organic Building Blocks of the Chemical Industry. John Wiley \& Sons, Hoboken, 522.

[7] Morais, G.R., Watanabe, M., Tanaka, Y. and Thiemann, T. (2005) Simple Amidation of Unprotected Phenol-Containing 2-Alkenoic Acids. Journal of Chemical Research, 29, 802. http://dx.doi.org/10.3184/030823405775146889

[8] Morais, G.R. and Thiemann, T. (2012) Synthesis of Estradiol-Cinnamide Conjugates. Journal of Chemical Research, 36, 549-554. http://dx.doi.org/10.3184/174751912X13419346932954

[9] Liu, L., Hudgins, W.R., Shack, S., Yin, M.Q. and Samid, D. (1995) Cinnamic Acid: A Natural Product with Potential Use in Cancer Intervention. International Journal of Cancer, 62, 345-350. http://dx.doi.org/10.1002/ijc.2910620319

[10] Mobinikhaledi, N., Foroughifar, N. and Fathinejad, J.H. (2008) Microwave-Assisted Synthesis of Cinnamic Acid Derivatives in the Presence of PPE and under Solvent-Free Conditions. Synthesis and Reactivity in Inorganic, Metal-Organic and Nano-Metal Chemistry, 38, 428-430.

[11] Gupta, M. and Wakhloo, B.P. (2007) Tetrabutylammonium Bromide Mediated Knoevenagel Condensation in Water: Synthesis of Cinnamic Acids. ARKIVOC, 15, 94-98.

[12] Zolfigol, M.A., Ayazi-Nasrabadi, R. and Baghery, S. (2015) Synthesis and Characterization of Two Novel Biological-Based Nano Organo Solid Acids with Urea Moiety and Their Catalytic Applications in the Synthesis of 4,4'(Arylme-Thylene)Bis(1H-Pyrazo-5-ol), Coumarin3-Carboxylic Acid, and Cinnamic Acid Derivatives under Mild and Green Conditions. RSC Advances, 5, 71942-71954. http://dx.doi.org/10.1039/C5RA14001C

[13] Johnson, J.R. (1942) Perkin Reaction and Related Reactions. Organic Reactions, 1, 210-265. http://doi:10.1002/0471264180.or001.08

[14] Carmichael, A.J., Earle, M.J., Holbrey, J.D., McCormac, P.B. and Seddon, K.R. (1999) The Heck Reaction in Ionic Liquids: A Multiphasic Catalyst System. Organic Letters, 1, 997-1000. http://doi:10.1021/o19907771

[15] Thiemann, T. (2007) Solventless Wittig Olefination with Fluorinated Benzaldehydes. Journal of Chemical Research, 6 , 336-341. http://dx.doi.org/10.3184/030823407X225464

[16] Thiemann, T., Thiemann, C., Sasaki, S., Vill, V., Mataka, S. and Tashiro, M. (1997) Synthesis and Photochemical Behavior of 3-(Estran-16-yl)acrylates and 2-(Estran-16-yl)vinyl Ketones. Journal of Chemical Research $(S)$, 248-249, 1736. http://dx.doi.org/10.1039/A700031F

[17] Al Jasem, Y., El-Esawi, R. and Thiemann, T. (2014) Wittig- and Horner-Wadsworth-Emmons-Olefination Reactions with Stabilized and Semi-Stabilized Phosphoranes and Phosphonates under Non-Classical Conditions. Journal of Chemical Research, 38, 453-463. http://dx.doi.org/10.3184/174751914X14044684705710

[18] Thiemann, T., Watanabe, M., Tanaka, Y. and Mataka, S. (2004) Solvent-Free Wittig Olefination with Stabilized Phosphoranes-Scope and Limitations. New Journal of Chemistry, 28, 578-584. http://dx.doi.org/10.1039/B311894K 
[19] Le Boulaire, V. and Gree, R. (2000) Wittig Reactions in the Ionic Solvent [bmim][BF 4 . Chemical Communications, 2195-2196. http://dx.doi.org/10.1039/b006666o

[20] Broos, R., Tavernier, D. and Ateunis, M. (1978) P-Carboxystyrene. A Wittig Procedure in Aqueous Medium. Journal of Chemical Education, 55, 813. http://dx.doi.org/10.1021/ed055p813

[21] Russell, M.G. and Warren, S. (1998) Wittig Reactions in Water. Synthesis of New Water-Soluble Phosphonium Salts and Their Reactions with Substituted Benzaldehydes. Tetrahedron Letters, 39, 7995-7998. http://dx.doi.org/10.1016/S0040-4039(98)01740-7

[22] Russell, M.G. and Warren, S. (2000) Synthesis of New Water-Soluble Phosphonium Salts and Their Wittig-Reactions in Water. Journal of the Chemical Society, Perkin Transactions 1, 505-513. http://dx.doi.org/10.1039/a909495d

[23] Butcher, M., Mathews, R.J. and Middleton, S. (1973) Synthesis of ar-Nitrostyrenes. Australian Journal of Chemistry, 26, 2067-2069. http://dx.doi.org/10.1071/CH9732067

[24] Broos, R. and Anteunis, M. (1976) A Simplified Wittig Synthesis of Substituted Styrenes. Synthetic Communications, 6, 53-57. http://dx.doi.org/10.1080/00397917608062133

[25] Schleich, K. and Stoller, H., Hoffmann La-Roche, Inc., Nutley, N.J. (1979) US Pat. 4,254,281. Appl. July 25 th, 1979.

[26] Dambacher, J., Zhao, W., El-Batta, A., Anness, R., Jiang, C. and Bergdahl, M. (2005) Water Is an Efficient Medium for Wittig Reactions Employing Stabilized Ylides and Aldehydes. Tetrahedron Letters, 46, 4473-4477. http://dx.doi.org/10.1016/j.tetlet.2005.04.105

[27] Wu, J., Li, D. and Zhang, D. (2005) Aqueous Wittig Reactions of Aldehydes with in Situ Formed. Synthetic Commununications, 35, 2543-2551. http://dx.doi.org/10.1080/00397910500213054

[28] Wu, J., Zhang, D. and Wei, S. (2005) Wittig Reactions of Stabilized Phosphorus Ylides in Water. Synthetic Communications, 35, 1213-1222. http://dx.doi.org/10.1081/SCC-200054816

[29] Wu, J. and Yue, C. (2006) One-Pot Wittig Reactions in Aqueous Media: A Rapid and Environmentally Benign Synthesis of $\alpha, \beta$-Unsaturated Carboxylic Esters and Nitriles. Synthetic Commununications, 36, 2939-2947. http://dx.doi.org/10.1080/00397910600773684

[30] Orsini, F., Sello, G. and Fumagalli, T. (2006) One-Pot Wittig Reactions in Water in Presence of a Surfactant. ChemInform, 37. http://dx.doi.org/10.1002/chin.200647094

[31] El-Batta, A., Jiang, C., Zhao, W., Anness, R., Cooksy, A.L. and Bergdahl, M. (2007) Wittig Reactions in Water Media Employing Stabilized Ylides with Aldehydes. Synthesis of $\alpha, \beta$-Unsaturated Esters from Mixing Aldehydes, $\alpha$-Bromoesters, and $\mathrm{Ph}_{3} \mathrm{P}$ in Aqueous $\mathrm{NaHCO}_{3}$. The Journal of Organic Chemistry, 72, 5244-5259. http://dx.doi.org/10.1021/jo070665k

[32] Molander, G.A. and Oliveira, R.A. (2008) Wittig Reactions of Formyl Substituted Organotrifluoroborates and Stabilized Phosphonium Ylides in an Aqueous Medium. Tetrahedron Letters, 49, 1266-1268. http://dx.doi.org/10.1016/j.tetlet.2007.11.205

[33] McNulty, J. and Das, P. (2009) Highly Stereoselective and General Synthesis of (E)-Stilbenes and Alkenes by Means of an Aqueous Wittig Reaction. European Journal of Organic Chemistry, 2009, 4031-4035. http://dx.doi.org/10.1002/ejoc.200900634

[34] Odinets, I.L. and Matveeva, E.V. (2010) Ionic Liquids and Water as “Green” Solvents in Organophosphorus Synthesis. Current Organic Chemistry, 14, 1171-1184. http://dx.doi.org/10.2174/138527210791330486

[35] Märkl, G. and Merz, A. (1973) Carbonyl-Olefinierungen mit nicht-stabilisierten Phosphin-alkylenen im wäßrigen System. Synthesis, 1973, 295-297. http://dx.doi.org/10.1055/s-1973-22193

[36] Watanabe, M., Morais, G.R., Mataka, S., Ideta, K. and Thiemann, T. (2005) Two Variations of Solvent-Reduced Wittig Olefination Reactions - Comparison of Solventless Wittig Reactions to Wittig Reactions under Ultrasonication with Minimal Work-Up. Zeitschrift für Naturforschung, 60B, 909-916.

[37] Tiwari, S. and Kumar, A. (2008) Unusual Temperature Dependence of Salt Effects for “on Water" Wittig Reaction: Hydrophobicity at the Interface. Chemical Communications, 37, 4445-4447. http://dx.doi.org/10.1039/b809127g

[38] Johnstone, R.A.W. and Rose, M.E. (1979) A Rapid, Simple and Mild Procedure for Alkylation of Phenols, Alcohols, Amides and Acids. Tetrahedron, 35, 2169-2173. http://dx.doi.org/10.1016/0040-4020(79)87035-0

[39] Considine, W.J. (1962) Triphenyl(Carbethoxymethyl)phosphonium Chloride Dihydrate. The Journal of Organic Chemistry, 27, 647-649. http://dx.doi.org/10.1021/jo01049a519

[40] Thiemann, T., Tanaka, Y., Ideta, K. and Mataka, S. (2006) Solvent-Reduced Wittig Olefination Reactions with Halo Containing Conjugated Phosphoranes. Central European Journal of Chemistry, 4, 403-427.

[41] Aldrich Catalogue 2005-2006.

[42] Hedvati, L., Nudelman, A., Falb, E., Kraiz, B., Zhuk, R. and Sprecher, M. (2002) Cinnamic Acid Derived Oxazolinium 
Ions as Novel Cytotoxic Agents. European Journal of Medicinal Chemistry, 37, 607-616. http://dx.doi.org/10.1016/S0223-5234(02)01375-2

[43] Khan, F.A., Ahmad, S., Kodipelli, N., Shivange, G. and Anindya, R. (2014) Synthesis of a Library of Molecules on the Marine Natural Product Ianthelliformisamines Platform and Their Biological Evaluation. Organic \& Biomolecular Chemistry, 12, 3847-3865. http://dx.doi.org/10.1039/c3ob42537a

[44] Wittmer, F.B. and Raiford, C.L. (1945) Oxidation of 3,4-Dimethoxycinnamic Acid and Substitution Products with Alkaline Potassium Permanganate Solution. The Journal of Organic Chemistry, 10, 527-532. http://dx.doi.org/10.1021/jo01182a006

[45] Saljoughian, M. and Williams, P.G. (1987) Using of Tritium and Deuterium in Assigning Proton NMR Spectra: Reinvestigation of the Configuration of 5-Phenylpenta-2,4-dienoic Acid (m.p. 165.Degree.). The Journal of Organic Chemistry, 52, 3481-3483. http://dx.doi.org/10.1021/jo00391a069

[46] Miller, R.E. and Nord, F.E. (1950) Studies in the Thiophene Series. VII. The Application of the Reformatsky Reaction to Thiophene Aldehydes and Ketones. The Journal of Organic Chemistry, 15, 89-95. http://dx.doi.org/10.1021/jo01147a015

[47] Ide, W.S. and Buck, J.S. (1937) Pharmacologically Active Compounds from Alkoxy- $\beta$-phenylethylamines. Journal of the American Chemical Society, 59, 726-731. http://dx.doi.org/10.1021/ja01283a038

[48] Mary, Y.S., Varghese, H.T., Panicker, C.Y., Thiemann, T., Al-Saadi, A.A., Popoola, S.A., van Alsenoy, C. and Al Jasem, Y. (2015) Molecular Conformational Analysis, Vibrational Spectra, NBO, NLO, HOMO-LUMO and Molecular Docking Studies of Ethyl 3-(E)-(anthracen-9-yl)prop-2-enoate Based on Density Functional Theory Calculations. Spectrochimica Acta Part A: Molecular and Biomolecular Spectroscopy, 150, 533-542. http://dx.doi.org/10.1016/j.saa.2015.05.092

[49] Arjunan, P., Shymasundar, N., Berlin, D.K., Najjar, D. and Rockley, M.G. (1981) Syntheses of Selected $\varepsilon-(2-$ or 9-Anthryl)alkanoic Acids and Certain Esters-Carbon-13 Spin-Lattice Relaxation Time Measurements of Methyl 5-(2-Anthryl)pentanoate and Methyl 7-(2-Anthryl)heptanoate. The Journal of Organic Chemistry, 46, 626-629. http://dx.doi.org/10.1021/jo00316a029

[50] Cho, J.-Y., Moon, J.-H., Seong, K.-Y. and Park, K.-H. (1998) Antimicrobial Activity of 4-Hydroxybenzoic Acid and trans 4-Hydroxycinnamic Acid Isolated and Identified from Rice Hull. Bioscience, Biotechnology and Biochemistry, 62, 2273-2276. http://dx.doi.org/10.1271/bbb.62.2273

[51] Abarbri, M., Thibonnet, J., Parrain, J.-L. and Duchêne, A. (2002) Palladium-Catalyzed Cross-Coupling of Iodovinylic Acids with Organometallic Reagents. Selective Synthesis of 3,3-Disubstituted Prop-2-enoic Acids. Synthesis, 2002, 543-551. http://dx.doi.org/10.1055/s-2002-20971

[52] Hauser, C.R. and Puterbaugh, W.H. (1953) Aldol Condensation of Esters with Ketones or Aldehydes from $\beta$-Hydroxy Esters by Lithium Amide. Comparison with the Reformatsky Reaction. Journal of the American Chemical Society, 75, 1068-1072. http://dx.doi.org/10.1021/ja01101a016

[53] Huang, H., Jia, K. and Chen, Y. (2015) Hypervalent Iodine Reagents Enable Chemoselective Deboronative/Decarboxylative Alkenylation by Photoredox Catalysis. Angewandte Chemie, International Edition in English, 54, 1881-1884.

[54] D’yakonov, L.A. and Komendatov, M.I. (1961) Reactions between Aliphatic Diazo-Compounds with Unsaturated Compounds. 20. Investigation of Reaction between Ethyl Ester of Daizoacetic Acid and 1-Phenylpropyne in Presence of Cupric Sulfate. Zhurnal Obshchei Khimii, 31, 3881-3881.

[55] Hurd, C.D. and Tockman, A. (1959) The cis and trans 12-Benzoyl-9,10-dihydro-9,10-ethanoanthracene-11-carboxylic Acids. Journal of the American Chemical Society, 81, 116-119. http://dx.doi.org/10.1021/ja01510a025

[56] Thota, N., Koul, S., Reddy, M.V., Sangwan, P.L., Khan, I.A., Kumar, A., Raja, A.F., Andotra, S.S. and Qazi, G.N. (2008) Citral Derived Amides as Potent Bacterial NorA Efflux Pump Inhibitors. Bioorganic \& Medicinal Chemistry, 16, 6535-6543. http://dx.doi.org/10.1016/j.bmc.2008.05.030

[57] Concellon, J.M. and Concellon, C. (2006) Direct Reaction of Dibromoacetic Acid with Aldehydes Promoted by Samarium Diiodide: An Easy, Efficient, and Rapid Synthesis of $(E)-\alpha, \beta$-Unsaturated Carboxylic Acids with Total Stereoselectivity. The Journal of Organic Chemistry, 71, 1728-1731. http://dx.doi.org/10.1021/jo052236x

[58] Kuang, C., Yang, Q., Senboku, H. and Tokuda, M. (2005) Synthesis of (Z)-1-bromo-1-alkenes and Terminal Alkynes from Anti-2,3-dibromoalkanoic Acids by Microwave Induced Reaction. Tetrahedron, 61, 4043-4052. http://dx.doi.org/10.1016/j.tet.2005.02.043

[59] Chenault, J. and Dupin, J.F.E. (1987) (Ethoxycarbonyliodomethyl)triphenylphosphonium Iodide: A Convenient Reagent for the Direct Synthesis of $\beta$-Substituted Propiolic Acids via the Corresponding Esters. Synthesis, 1987, 498-499. http://dx.doi.org/10.1055/s-1987-27983

[60] Newman, M.S. and Merrill, S.H. (1955) Synthesis of a Series of Substituted Phenylpropiolic Acids. Journal of the American Chemical Society, 77, 5549-5551. http://dx.doi.org/10.1021/ja01626a033 
[61] Bonnin, C.M., Cadby, P.A., Freeman, C.G. and Ward, A.D. (1979) Acetylenic Acids IV. The Reactions of Alkoxy-Substituted Phenylpropiolic Acids and Esters with Sulfur Halides, Particularly Thionyl Chloride. Australian Journal of Chemistry, 32, 833-847. http://dx.doi.org/10.1071/CH9790833

[62] Rowland, A.T. (2002) CER Laboratory Program in Chemistry. Jeffers, J., Ed., REAC 750, Wadsworth Group.

[63] Bergmann, E.D., Ginsburg, D. and Lavie, D. (1950) Aryltrichloromethylcarbinols. Journal of the American Chemical Society, 72, 5012-5014. http://dx.doi.org/10.1021/ja01167a051

[64] Nguyen, K.C. and Weizman, H. (2007) Greening Wittig Reactions: Solvent-Free Synthesis of Ethyl trans-Cinnamate and trans-3-(9-Anthranyl)-2-propenoate. Journal of Chemical Education, 84, 119. http://dx.doi.org/10.1021/ed084p119

[65] Yin, J., Li, Y., Zhang, R., Jin, K. and Duan, C. (2014) Copper/Silver-Mediated Decarboxylative Trifluoromethylation of $\alpha, \beta$-Unsaturated Carboxylic Acids with $\mathrm{CF}_{3} \mathrm{SO}_{2} \mathrm{Na}$. Synthesis, 46, 607-612. http://dx.doi.org/10.1055/s-0033-1338578

[66] Rossi, D., Urbano, M., Baraglia, A., Carnevale, S.M., Bergamelli, F., Cannellini, M., Azzolina, O. and Collina, S. (2009) Polymer-Assisted Solution-Phase Synthesis under Combined Ultrasound and Microwave Irradiation: Preparation of $\alpha, \beta$-Unsaturated Esters and Carboxylic Acids, Key Intermediates of Novel Sigma Ligands. Synthetic Communications, 39, 3254-3262. http://dx.doi.org/10.1080/00397910902738112

[67] Liang, B., Dai, M., Chen, J. and Yang, Z. (2005) Copper-Free Sonogashira Coupling Reaction with $\mathrm{PdCl}_{2}$ in Water under Aerobic Conditions. The Journal of Organic Chemistry, 70, 391-393. http://dx.doi.org/10.1021/jo048599z

[68] Li, Y., Lu, L.-Q., Das, S., Pisiewicz, S., Junge, K. and Beller, M. (2012) Highly Chemoselective Metal Free of Phosphine Oxides to Phosphines. Journal of the American Chemical Society, 134, 18325-18329. http://dx.doi.org/10.1021/ja3069165

[69] Li, Y.-H., Das, S., Zhou, S.L., Junge, K. and Beller, M. (2012) General and Selective Copper-Catalyzed Reduction of Tertiary and Secondary Phosphine Oxides: Convenient Synthesis of Phosphines. Journal of the American Chemical Society, 134, 9727-9732. http://dx.doi.org/10.1021/ja301764m

[70] Petit, C., Poli, E., Favre-Reguillon, A., Khrouz, L., Denis-Quanquin, S., Bonneviot, L., Mignani, G. and Lemaire, M. (2013) Unraveling the Catalytic Cycle of Tertiary Phosphine Oxides Reduction with Hydrosiloxane and $\mathrm{Ti}(\mathrm{O} i \mathrm{Pr})_{4}$ through EPR and ${ }^{29} \mathrm{Si}$ NMR Spectroscopy. ACS Catalysis, 3, 1431-1438. http://dx.doi.org/10.1021/cs4002767

[71] Imamoto, T., Takeyama, T. and Kusumoto, T. (1985) Facile Reduction of Organic Halides and Phosphine Oxides with $\mathrm{LiAlH}_{4}-\mathrm{CeCl}_{3}$. Chemistry Letters, 14, 1491-1492. http://dx.doi.org/10.1246/cl.1985.1491

[72] Pommer, H. and Thieme, P.C. (2005) Industrial Application of the Wittig Reaction. In: Houk, K.N., Eds., Wittig Chemistry, Springer, Berlin, 165-188. http://dx.doi.org/10.1007/BFb0018058 